\title{
Block-iterative generalized decision feedback equalizers for large MIMO systems: algorithm design and asymptotic performance analysis
}

Citation for published version (APA):

Liang, Y-C., Sun, S., \& Ho, C. K. (2006). Block-iterative generalized decision feedback equalizers for large MIMO systems: algorithm design and asymptotic performance analysis. IEEE Transactions on Signal Processing, 54(6), 2035-2048. https://doi.org/10.1109/TSP.2006.873485

DOI:

10.1109/TSP.2006.873485

Document status and date:

Published: 01/01/2006

Document Version:

Publisher's PDF, also known as Version of Record (includes final page, issue and volume numbers)

Please check the document version of this publication:

- A submitted manuscript is the version of the article upon submission and before peer-review. There can be important differences between the submitted version and the official published version of record. People interested in the research are advised to contact the author for the final version of the publication, or visit the $\mathrm{DOI}$ to the publisher's website.

- The final author version and the galley proof are versions of the publication after peer review.

- The final published version features the final layout of the paper including the volume, issue and page numbers.

Link to publication

\footnotetext{
General rights

- You may freely distribute the URL identifying the publication in the public portal. follow below link for the End User Agreement:

www.tue.nl/taverne

Take down policy

If you believe that this document breaches copyright please contact us at:

openaccess@tue.nl

providing details and we will investigate your claim.
}

Copyright and moral rights for the publications made accessible in the public portal are retained by the authors and/or other copyright owners and it is a condition of accessing publications that users recognise and abide by the legal requirements associated with these rights.

- Users may download and print one copy of any publication from the public portal for the purpose of private study or research.

- You may not further distribute the material or use it for any profit-making activity or commercial gain

If the publication is distributed under the terms of Article $25 \mathrm{fa}$ of the Dutch Copyright Act, indicated by the "Taverne" license above, please 


\title{
Block-Iterative Generalized Decision Feedback Equalizers for Large MIMO Systems: Algorithm Design and Asymptotic Performance Analysis
}

\author{
Ying-Chang Liang, Senior Member, IEEE, Sumei Sun, and Chin Keong Ho, Student Member, IEEE
}

\begin{abstract}
This paper studies the problem of signal detection for multiple-input multiple-output (MIMO) channels with large signal dimensions. We propose a block-iterative generalized decision feedback equalization (BI-GDFE) receiver to recover the transmitted symbols in a block-iterative manner. By exploiting the input-decision correlation, a measure for the reliability of the earlier-made decisions, we design the feed-forward equalizers (FFEs) and feedback equalizers (FBEs) in such a way that maximized signal-to-interference-plus-noise ratio (SINR) is achieved for each of the iterations. Novel implementations are also introduced to simplify the complexity of the receiver, which requires only one-tap filters for FFE and FBE. The proposed receiver also works when the signal dimension is greater than the observation dimension. The asymptotic performance of the proposed receiver is analyzed and its convergence has been confirmed through numerical evaluations for various parameters. Computer simulations are presented to illustrate the capability of the proposed receiver to achieve single user matched-filter bound (MFB) for large random MIMO channels when the received SNR is high enough.
\end{abstract}

Index Terms-Asymptotic performance, generalized decision feedback equalization (GDFE), iterative receiver, multiple-input multiple-output (MIMO), signal detection.

\section{INTRODUCTION}

$\mathbf{I}$ $\mathrm{N}$ this paper, we are concerned with the problem of signal detection for the following generic multiple-input multipleoutput (MIMO) channel

$$
\begin{aligned}
\mathbf{x} & =\mathbf{H} \mathbf{s}+\mathbf{n} \\
& =\sum_{i=1}^{P} \mathbf{h}_{i} s(i)+\mathbf{n}
\end{aligned}
$$

where $\mathbf{s}=[s(1), s(2), \ldots, s(P)]^{\mathrm{T}}$ denotes the transmitted signal vector of dimension $P \times 1 ; \mathbf{h}_{i}$ represents the channel vector of dimension $N \times 1$, corresponding to symbol $s(i)$; $\mathbf{X}$ and $\mathbf{n}$ denote the received signal vector, and received noise vector, respectively, both with dimension $N \times 1 ; \mathbf{H}=\left[\mathbf{h}_{1}, \mathbf{h}_{2}, \ldots, \mathbf{h}_{P}\right]$

Manuscript received January 18, 2005; revised July 27, 2005. A portion of this work was presented at the International Conference on Communication, Seoul, Korea, May 2005. The associate editor coordinating the review of this manuscript and approving it for publication was Dr. Sergios Theodoridis.

Y.-C. Liang and S. Sun are with Institute for Infocomm Research, Singapore 119613 (e-mail: ycliang@i2r.a-star.edu.sg; sunsm@i2r.a-star.edu.sg).

C. K. Ho was with the Institute for Infocomm Research, Singapore 119613. $\mathrm{He}$ is now with the Department of Electrical Engineering, Eindhoven University of Technology, 5600 MB Eindhoven, The Netherlands (e-mail: c.k.ho@tue.nl).

Digital Object Identifier 10.1109/TSP.2006.873485 is the $N \times P$ channel matrix. In (1), $P$ and $N$ are referred to as signal dimension and observation dimension, respectively. The input-output model of (1) can be derived either in time, frequency, space, or code domain, or any combinations of them. We are interested in large systems $(P$ and $N$ are both large), typical examples of which include single carrier cyclic prefix (SCCP) systems [18], cyclic prefix based code division multiple access (CP-CDMA), multicarrier CDMA (MC-CDMA), MIMO/SCCP [19], [20], MIMO/MC-CDMA, MIMO/CP-CDMA systems, and pure MIMO antenna systems [14] with large antenna sizes. Large systems can also be found in wired transmissions. For example, in digital subscriber line (DSL) communications, it is common that hundreds of telephone lines are bounded together, thus there exists severe crosstalk, and the signal separation becomes very challenging because of the large signal dimension.

Linear and nonlinear receivers have been proposed to recover the transmitted symbols. Linear receivers, such as zero-forcing (ZF) and minimum mean square error (MMSE) equalizers, and some nonlinear receivers, such as VBLAST receiver, or so called generalized decision feedback equalizer (GDFE) [13], are simple to implement, however, the achievable performance is usually far away from the maximum likelihood (ML) performance bound. When the signal dimension is very small, exhaustive search can be used to achieve the ML detection. For large signal dimensions, however, the exponential complexity of the ML detector makes it generally impractical for implementation. Recently, a lot of efforts have been devoted in designing low-complexity nonlinear receivers to achieve near-ML performance for MIMO systems. When the signal dimension is small and moderate, near ML detection can be achieved through closest lattice point search (CPS) techniques [1], such as sphere decoding [3], [4], [2], with much lower complexity than brute force ML detector. The computational complexity and memory requirement of CPS techniques grow dramatically with the increase of the signal dimension. Thus, it becomes impractical again to implement CPS when the signal dimension goes large.

A block-iterative DFE (BI-DFE) receiver has been proposed for intersymbol interference (ISI) and multiple access interference (MAI) cancellation in CDMA systems in [5], and for ISI channel equalization in [6], assuming the availability of infinite signal dimension. This receiver iteratively estimates the 
transmitted symbols, and uses these decision-directed symbols to obtain a new set of symbol estimates by cancelling out the interferences. As an iterative technique, for high SNR region and when the number of independent delay paths is sufficiently large, BI-DFE ia able to achieve the single user matched filter bound (MFB). BI-DFE was extended to frequency domain equalization for SCCP systems in [7], and to layered space-time processing for multiuser SCCP systems in [20].

In this paper, building on the existing BI-DFE receiver, we propose a low-complexity iterative receiver for MIMO channels with large signal dimensions, called large MIMO systems. Our technique is a block-iterative generalized decision feedback equalizer (BI-GDFE), which detects the transmitted symbols in a block-iterative fashion. The coefficients of the input-decision correlation (IDC), a measure for the reliability of the earlier-made decisions relative to the transmitted symbols, are utilized to design the feed-forward equalizers (FFEs) and feedback equalizers (FBEs) in such a way that maximized signal-to-interference-plus-noise ratio (SINR) is achieved for each iteration. Novel implementations are also proposed to simplify the receiver complexity by making use of the singular value decomposition (SVD) of the MIMO channel matrix.

We study the proposed BI-GDFE receiver from two perspectives: signal detection for MIMO systems with finite signal dimensions and asymptotic performance analysis for large random MIMO channels.

For MIMO systems with finite signal dimensions, BI-GDFE receiver is in general sensitive to the selection of the IDC coefficients. If the IDCs are chosen improperly, the algorithm may not converge to the optimal solution, or may have very slow convergence. In [5] and [6], the theoretical IDC formula for ISI channels is derived for PSK modulations, assuming the availability of infinite signal dimension. For finite dimension systems, the IDC coefficients are in fact random variables fluctuating from one realization to another. Thus, the IDC selection method of [5] and [6] cannot be applied in most of the practical systems. In this paper, a method is proposed to determine the IDC coefficients with which fast and guaranteed convergence can be achieved for the proposed BI-GDFE receiver.

The asymptotic performance of the proposed BI-GDFE for large random MIMO channels is analyzed based on the pioneering work conducted by Tse and Hanly [11], Verdú and Shamai [12]. According to [11] and [12], while the coefficients of the MMSE receiver are dependent on the channel coefficients, the output SINR of the MMSE receiver for random MIMO channels tends to be a constant value, which is determined by the average received SNRs of all users, as well as the traffic load $P / N$, when $P \rightarrow \infty, N \rightarrow \infty$, and $P / N \rightarrow$ constant $\alpha$. The available results are utilized to analyze the limiting performance of the proposed BI-GDFE receiver for random MIMO systems. Specifically, we derive the limiting SINRs for the proposed BI-GDFE for each of the iterations, and illustrate through numerical results the convergence of the limiting SINR to the single user received SNR when the received SNR is sufficiently high.

This paper is organized as follows. Section II presents the generic MIMO channel model, and reviews the conventional linear and nonlinear equalizers. In Section III, we propose the
BI-GDFE receiver, and obtain the optimal FFE and FBE for each of the iterations. Section IV studies the issue of IDC selection, which plays an important role for the proposed receiver. Suboptimal BI-GDFE algorithms are presented in Section V to simplify the calculations. The asymptotic performance of the proposed BI-GDFE receiver for large random MIMO channels is analyzed in Section VI. Computer simulations are given in Section VII, and, finally, conclusions are drawn in Section VIII.

\section{CHANnEl Model AND CONVENTIONAL RECEIVERS}

\section{A. Channel Model}

The channel model considered in this paper follows the inputoutput relation as shown in (1), which can be derived either in time, frequency, space, or code domain, or any combinations of them. We list some of the systems which fit into that model.

1) MIMO Spatial Multiplexing Systems: A communication system with $n_{t}$ transmitters and $n_{r}$ receivers is referred as a $\left(n_{t}, n_{r}\right)$ MIMO antenna system. If the channel coefficients are statistically independent, a $\left(n_{t}, n_{r}\right)$ MIMO antenna system achieves a channel capacity almost linearly proportional to $\min \left(n_{t}, n_{r}\right)$. In order to achieve this capacity limit, the spatial multiplexing scheme has been proposed, which consists of transmitting independent data streams through different transmit antennas. Due to the cross-interference, each receive antenna receives a combination of the transmitted symbols, which have to be separated at the receiver side using signal processing techniques. For a MIMO antenna spatial multiplexing system operating in a flat fading environment, the channel matrix $\mathbf{H}$ represents the channel responses from each of the transmit antennas to each of the receive antennas. Thus $s(i)$ in (2) represents the data symbol sent through the $i$ th transmit antenna, and we have $P=n_{t}$, and $N=n_{r}$.

2) CDMA Systems: For frequency-flat synchronous CDMA uplink, we are interested in the received signals at the base station. Let $s(i)$ in (2) represent the data symbol for the $i$ th user, and $\mathbf{h}_{i}=\alpha_{i} \mathbf{c}_{i}$, where $\alpha_{i}$ denotes the channel gain the user $i$ to base station, and $\mathbf{c}_{i}$ as the spreading code sequence of dimension $N \times 1$ for user $i$. Thus, the channel matrix represents the composite effect of channel responses and user-dependent spreading codes, and it becomes a random matrix when the spreading codes are chosen to be random, which is the case for third generation wideband CDMA systems. Thus, $P$ denotes the number of users, and $N$ represents the spreading factor.

3) SCCP Systems: For frequency selective channels, blockbased transmissions can be used to compensate for the delay spread using computationally efficient receivers. For a SCCP system [18], each block of $N_{c}$ symbols is pre-inserted with a $\mathrm{CP}$ portion longer than the the maximum excess delay of the channel. At the receiver side, the received portion corresponding to the CP part is discarded, and the $N_{c}$ received signals corresponding to the rest part of each block can be put into a vector format, which can be represented by (1). Because of the adding of $\mathrm{CP}$, the time domain channel matrix becomes a circulant matrix, which can be decomposed as $\mathrm{W}^{\mathrm{H}} \Lambda \mathbf{W}$, where $\mathbf{W}$ is the $N_{c}$-point DFT matrix, and $\boldsymbol{\Lambda}$ is a diagonal matrix, the $i$ th element of the diagonal components representing the frequency domain channel response at the $i$ th frequency tone. If we treat 
the DFT output of each block as the observation block, then the channel matrix becomes $\mathbf{H}=\Lambda \mathbf{W}$. Thus in this case, $P=N=N_{c}$.

For a MIMO system working in a frequency selective environment, we can incorporate SCCP and spatial multiplexing schemes [19], [20]. In this case, we have $P=n_{t} N_{c}$ and $N=$ $n_{r} N_{c}$, where $n_{t}$ and $n_{r}$ are the number of transmit antenna and receive antenna, respectively.

The model (1) can also be used to represent systems like CP-CDMA, MC-CDMA, MIMO/CP-CDMA, and MIMO/MCCDMA. Further, the transmitted signal vector $\mathbf{s}$ may consist of the information-bearing symbols coming from the same user, which are usually with the same type of modulation. For multiuser communications, each element of $\mathbf{s}$ may represent different types of modulations. Because of the generality of the channel model (1), our proposed algorithms can be applied to any of those systems. Finally, in the channel model (2), the signal dimension $P$ can be greater than the observation dimension $N$, which means, for MIMO antenna systems, each of the transmit antennas can send an independent data stream, even for the case of $n_{t}>n_{r}$. This is not a problem in terms of receiver design to recover the transmitted symbols as the system may be operating below the channel capacity, especially when BPSK or QPSK is applied. We will provide the simulation results for such scenario in Section VII.

Throughout this paper, we make the following assumptions.

AS1) The channel responses $\mathbf{h}_{k}$ is represented as $\mathbf{h}_{k}=(1 / \sqrt{N})\left[h_{1, k}, h_{2, k}, \ldots, h_{N, k}\right]^{\mathrm{T}}$, where $\mathrm{E}\left[\left|h_{j, k}\right|^{2}\right]=1$.

AS2) The transmitted symbols $s(i)$ s are zero mean, iid and with power $\mathrm{E}\left[|s(i)|^{2}\right]=\sigma_{s}^{2}$.

AS3) The noise vector $\mathbf{n}$ is zero mean, circularly symmetric, complex Gaussian with covariance matrix $\mathrm{E}\left[\mathbf{n n}^{\mathrm{H}}\right]=$ $\sigma_{n}^{2} \mathbf{I}$.

We define $\gamma=\sigma_{s}^{2} / \sigma_{n}^{2}$ as the average received signal-to-noise ratio (SNR). For MIMO antenna systems, the readers are cautioned to be careful since for spatial multiplexing, it is conventionally defined that the total transmission power is fixed, thus transmission power is split over the transmission data streams. Here, with the new normalization, we define the average received power of each data stream over all receive antennas. Thus, the SNR is defined as the average received SNR.

\section{B. Conventional Equalizers}

Let us first consider the linear receivers. Denote $\mathbf{K}=$ $\left[\mathbf{k}_{1}, \mathbf{k}_{2}, \ldots, \mathbf{k}_{P}\right]$ as the weight matrix for the $P$ transmitted symbols. The SINR of the soft decision $\hat{s}(i)=\mathbf{k}_{i}^{\mathrm{H}} \mathbf{x}$ for determining $s(i)$ is given by

$$
\beta_{i}=\frac{\left|\mathbf{k}_{i}^{\mathrm{H}} \mathbf{h}_{i}\right|^{2}}{\sum_{j \neq i}\left|\mathbf{k}_{i}^{\mathrm{H}} \mathbf{h}_{j}\right|^{2}+\left(\frac{1}{\gamma}\right)\left\|\mathbf{k}_{i}\right\|^{2}}
$$

The zero-forcing $(\mathrm{ZF})$ receiver is given by

$$
\mathbf{K}_{\mathrm{ZF}}=\left(\mathbf{H H}^{\mathrm{H}}\right)^{-1} \mathbf{H} \text {. }
$$

Note the ZF equalizer tries to null out the interferences from the other users/symbols, and it usually suffers from noise enhance- ment because of the nulling purpose. Note the inverse should be replaced by pseudoinverse when $N>P$.

The single user matched filter (SUMF) equalizer is given by $\mathbf{K}_{\mathrm{SUMF}}=\mathbf{H}$ which maximizes the power gain at the desired symbols' directions, but ignores the existence of the other symbols coming from the other directions. As such, SUMF suffers from strong interferences from the other symbols.

The MMSE receiver is given by

$$
\mathbf{K}_{\mathrm{MMSE}}=\left(\mathbf{H} \mathbf{H}^{\mathrm{H}}+\left(\frac{1}{\gamma}\right) \mathbf{I}\right)^{-1} \mathbf{H} \text {. }
$$

As the optimal linear receiver, MMSE receiver maximizes the output SINR by compromising the interference nulling and noise enhancement. The output SINR for symbol $s(i)$ is given by

$$
\beta_{i}=\mathbf{h}_{i}^{\mathrm{H}}\left(\mathbf{H}_{i} \mathbf{H}_{i}^{\mathrm{H}}+\left(\frac{1}{\gamma}\right) \mathbf{I}\right)^{-1} \mathbf{h}_{i}
$$

where $\mathbf{H}_{i}$ is the matrix $\mathbf{H}$ with the $i$ th column deleted. For random MIMO channels, according to random matrix theory, even though the MMSE weight matrix (4) is dependent on the channel coefficients, the output SINR in (5) converges to a fixed value as $P \rightarrow \infty, N \rightarrow \infty$, and $P / N \rightarrow \alpha$, where $\alpha$ is a constant [11]. We will get back to this point in more details in Section VI.

Finally, the GDFE receiver, or called VBLAST receiver [13], successively performs nulling-based detection and interference cancellation. The nulling-based detection makes use of MMSE receiver or $\mathrm{ZF}$ receiver. The interference cancellation operation then cancels out the interferences using earlier-made decisions. Similarly to conventional DFE, the GDFE receiver is very sensitive to propagation of decision errors generated in the earlier stages.

\section{BI-GDFE}

In this section, we propose a BI-GDFE receiver for large MIMO systems. The idea behind the proposed receiver is to estimate the transmitted symbols of each block in a "block-iterative" manner. Specifically, for the given observation vector $\mathbf{x}$, we estimate each of transmitted symbols using MMSE receiver, and make hard decisions for all the symbols. This is called one iteration. Then the decisions made at the previous iteration are utilized to cancel out the interferences corresponding to each of the symbols to be detected further.

We use subscript $\ell_{\ell}$ to denote the $\ell$ th iteration. The BI-GDFE makes use of the decision-directed symbols from the $(\ell-1)$ th iteration to assist for signal detection for the $\ell$ th iteration. With reference to Fig. 1, at the $\ell$ th iteration, the received signal $\mathbf{x}$ is passed to a FFE, $\mathbf{K}_{\ell}^{\mathrm{H}}$; at the same time, the hard decisions, $\hat{\mathbf{s}}_{\ell-1}$, generated at the $(\ell-1)$ th iteration go through a FBE, $\mathbf{D}_{\ell}$. The outputs from the two equalizers are then combined, and further utilized to generate hard decisions for the $\ell$ th iteration. Denote $\mathbf{z}_{\ell}$ as the combiner output:

$$
\mathbf{z}_{\ell}=\mathbf{K}_{\ell}^{\mathrm{H}} \mathbf{x}+\mathbf{D}_{\ell} \hat{\mathbf{s}}_{\ell-1} .
$$

The key idea of BI-GDFE is to design the FFE and FBE in such a way that the signal component $\mathrm{s}$ contained in the observation 


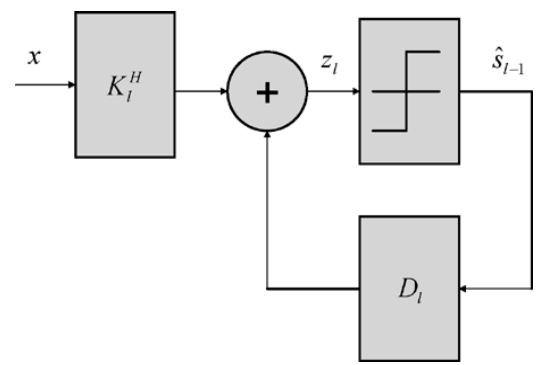

Fig. 1. Block diagram of the proposed BI-GDFE receiver for generic MIMO systems.

vector $\mathbf{x}$ is preserved and recovered by the FFE, while the interferences are cancelled out, using the FBE, as much as possible.

Before proceeding to deriving the optimal FFE and FBE for each of the iterations, let us first introduce the concept of IDC.

Definition 1: The correlation matrix between transmitted symbol vector, $\mathbf{s}$, and decision symbol vector, $\hat{\mathbf{s}}_{\ell}$, generated at the $\ell$ th iteration is given by

$$
\mathrm{E}\left[\mathbf{s} \hat{\mathbf{s}}_{\ell}^{\mathrm{H}}\right]=\rho_{\ell} \sigma_{s}^{2} \mathbf{I}
$$

where $\rho_{\ell}=\left(1 / \sigma_{s}^{2}\right) \mathrm{E}\left[s(i) \hat{s}_{\ell}^{*}(i)\right]$ is referred to as IDC.

The IDC reflects the statistical reliability of the decision-directed symbols as compared to the transmitted symbols when the block size $P$ goes to infinity. Thus it is independent of the symbol index $i$. Further, the IDC closely relates to the symbol/bit error rate (SER/BER) of the system. For an AWGN channel with QPSK modulation and an SNR of $\gamma_{0}$, we have that [6],

$$
\rho=1-2 P_{e}\left(\gamma_{0}\right)
$$

where $P_{e}\left(\gamma_{0}\right)=Q\left(\sqrt{\gamma_{0}}\right)$ [10] with $Q(u)=(1 / \sqrt{2 \pi})$ $\int_{u}^{\infty} e^{-t^{2} / 2} d t$. This can be verified as follows. Suppose $s(i)=(1+j)$ is transmitted, the probabilities for which $\hat{s}(i)=(1+j), \hat{s}(i)=(1-j), \hat{s}(i)=(-1+j)$, and $\hat{s}(i)=(-1-j)$ are, respectively, equal to $\left(1-P_{e}\left(\gamma_{0}\right)\right)^{2}$, $P_{e}\left(\gamma_{0}\right), P_{e}\left(\gamma_{0}\right)$, and $P_{e}\left(\gamma_{0}\right)^{2}$. Thus (8) follows immediately by considering that $s(i)$ can choose each of the four constellation points $( \pm 1 \pm j)$ with equal probability. Note the IDC increases when the SNR increases, and it will be upper-bounded by 1, which happens when all transmitted symbols are correctly recovered $\left(P_{e}\left(\gamma_{0}\right)=0\right)$. Finally, in the receiver design, we wish $\rho_{\ell} \rightarrow 1$ when $\ell \rightarrow \infty$ such that $\hat{\mathbf{s}}_{\ell} \rightarrow \mathbf{s}$.

Similar to [6], we make the following assumption:

AS4) $\hat{s}_{\ell}(i)$ s are zero-mean, iid variables, with equal energy, $\sigma_{s}^{2}$.

The iid assumption for the detected symbols is true when all transmitted symbols are correctly estimated, since the transmitted symbols have been assumed to be iid. Here we will analyze the case when the transmitted symbols may not be perfectly estimated when the SNR is not high enough and a practical MMSE receiver is used.

Rigorous proof for this assumption can be derived for large random channels $(P \rightarrow \infty, N \rightarrow \infty$ and $P / N \rightarrow$ constant $\alpha)$ using random matrix theory. Without loss of generality, we are interested in the detection of symbols $s(1)$ and $s(2)$. Specifically, from MIMO channel (1), the MMSE equalizer for detecting symbol $s(1)$ is given by

$$
\mathbf{k}_{1}=\left[\mathbf{H}_{1} \mathbf{H}_{1}^{\mathrm{H}}+\left(\frac{1}{\gamma}\right) \mathbf{I}\right]^{-1} \mathbf{h}_{1},
$$

where $\mathbf{H}_{1}$ is the matrix of $\mathbf{H}$ with the first column deleted; and the output of this equalizer is given by

$$
y(1)=\mathbf{k}_{1}^{\mathrm{H}} \mathbf{x}=\mathbf{k}_{1}^{\mathrm{H}}[\mathbf{H} s+\mathbf{n}] .
$$

Similarly, the MMSE equalizer, $\mathbf{k}_{2}$, and equalization output, $y(2)$, for detecting symbol $s(2)$ can be derived accordingly. Thus the cross-correlation between the equalization outputs, $y(1)$ and $y(2)$, of symbols $s(1)$ and $s(2)$ is given by

$$
\begin{aligned}
r_{12}= & \mathrm{E}\left[y(1) y^{*}(2)\right]=\mathbf{k}_{1}^{\mathrm{H}}\left[\mathbf{H} \mathbf{H}^{\mathrm{H}}+\left(\frac{1}{\gamma}\right) \mathbf{I}\right] \mathbf{k}_{2} \\
= & \mathbf{h}_{1}^{\mathrm{H}}\left[\mathbf{H}_{1} \mathbf{H}_{1}^{\mathrm{H}}+\left(\frac{1}{\gamma}\right) \mathbf{I}\right]^{-1}\left[\mathbf{H H}^{\mathrm{H}}+\left(\frac{1}{\gamma}\right) \mathbf{I}\right] \\
& \times\left[\mathbf{H}_{2} \mathbf{H}_{2}^{\mathrm{H}}+\left(\frac{1}{\gamma}\right) \mathbf{I}\right]^{-1} \mathbf{h}_{2} .
\end{aligned}
$$

In Appendix A, using random matrix theory, we show that the above correlation becomes zero for large random systems. Since the decisions $\hat{s}(1)$ and $\hat{s}(2)$ are based on $y(1)$ and $y(2)$, respectively, we conclude that the decisions are also iid for large random systems.

Similarly, we have the following results for the cross correlations between the $s(i)$ and $y(k)$

$$
\begin{aligned}
& \mathrm{E}\left[s(i) y^{*}(i)\right] \\
& =\sigma_{s}^{2} \mathbf{h}_{i}^{\mathrm{H}}\left(\mathbf{H}_{i} \mathbf{H}_{i}^{\mathrm{H}}+\left(\frac{1}{\gamma}\right) \mathbf{I}\right)^{-1} \mathbf{h}_{i} \rightarrow \sigma_{s}^{2} \beta, i . p . \text { for all } i \\
& \mathrm{E}\left[s(i) y^{*}(k)\right] \\
& =\sigma_{s}^{2} \mathbf{h}_{i}^{\mathrm{H}}\left(\mathbf{H}_{k} \mathbf{H}_{k}^{\mathrm{H}}+\left(\frac{1}{\gamma}\right) \mathbf{I}\right)^{-1} \mathbf{h}_{k} \rightarrow 0, i . p . \text { for } i \neq k .
\end{aligned}
$$

When the signal dimension is limited, it is in general difficult to prove AS4), however, our simulations (not included here due to space limitation) have shown that the correlations are actually close to zero. This is mainly due to the fact that $\hat{s}_{\ell}(i) \mathrm{s}$ are the hard decisions of slicer input, rather than the input itself.

The following theorem serves as the basis for the theoretical development of this paper.

Theorem 1: Consider the MIMO channel model (1), and assume that AS2)-AS4) are true. Let $\mathbf{A}_{\ell}=\operatorname{diag}\left(\mathbf{K}_{\ell}^{\mathrm{H}} \mathbf{H}\right)$, which takes the diagonal elements of $\mathbf{K}_{\ell}^{\mathrm{H}} \mathbf{H}$, then the optimal FFE and FBE maximizing the SINR at the slicer input at the $\ell$ th iteration are given by

$$
\begin{aligned}
& \mathbf{K}_{\ell}=\left[\left(1-\rho_{\ell-1}^{2}\right) \mathbf{H H}^{\mathrm{H}}+\left(\frac{1}{\gamma}\right) \mathbf{I}\right]^{-1} \mathbf{H} \\
& \mathbf{D}_{\ell}=\rho_{\ell-1}\left(\mathbf{A}_{\ell}-\mathbf{K}_{\ell}^{\mathrm{H}} \mathbf{H}\right)
\end{aligned}
$$

and the maximum SINR for the $i$ th symbol of $\mathbf{s}$ is given by

$$
\gamma_{\ell}(i)=\frac{\left|\mathbf{A}_{\ell}(i, i)\right|^{2} \sigma_{s}^{2}}{\mathbf{R}_{\tilde{n}_{\ell}}(i, i)}
$$


where $\mathbf{X}(i, j)$ denotes the element of the matrix $\mathbf{X}$ at the $i$ th row and $j$ th column, and

$$
\mathbf{R}_{\tilde{n}_{\ell}}=\frac{\sigma_{s}^{2}\left(1-\rho_{\ell-1}^{2}\right)}{\rho_{\ell-1}^{2}} \mathbf{D}_{\ell} \mathbf{D}_{\ell}^{\mathrm{H}}+\sigma_{n}^{2} \mathbf{K}_{\ell}^{\mathrm{H}} \mathbf{K}_{\ell} .
$$

Proof: See Appendix B.

The receiver algorithm based on Theorem 1 is referred to as Algorithm 1. Several comments are in order.

- The diagonal elements of the FBE $\mathbf{D}_{\ell}$ are all zeros, thus the previous decisions, $\hat{\mathbf{s}}_{\ell-1}$, are only used for the purpose of interference cancellation, and will not affect the signal components contained at the slicer input $\mathbf{z}_{\ell}$;

- The selection of the IDC coefficients plays a critical role to guarantee the convergence of the iterative algorithm. For the initialization, we set $\rho_{0}=0$, thus the FFE $\mathbf{K}_{1}$ is actually the conventional MMSE equalizer, and there is no feedback available. On the other hand, when the previously made decisions are perfect, the IDC coefficient is 1, thus the FBE cancels out all the interferences, and the FFE becomes SUMF, $(1 / \gamma) \mathbf{H}$.

Next, we are interested in the relations between the FFEs and FBEs of two different MIMO channels, whose transmitted signal vector and noise vectors are the same, but the channel matrices are related by a unitary matrix. The property of the matrix channel [16] can be be utilized.

Theorem 2: Consider the following two MIMO channels:

$$
\begin{aligned}
& \mathrm{CH} 1: \mathbf{x}_{1}=\mathbf{H}_{0} \mathbf{s}+\mathbf{n}_{1} \\
& \mathrm{CH} 2: \mathbf{x}_{2}=\mathbf{H} \mathbf{s}+\mathbf{n}_{2} .
\end{aligned}
$$

Suppose the channel matrix $\mathbf{H}$ can be decomposed as: $\mathbf{H}=$ $\mathbf{H}_{0} \mathbf{E}^{\mathrm{H}}$, where matrix, $\mathbf{E}$, is a unitary matrix, i.e., $\mathbf{E}^{\mathrm{H}} \mathbf{E}=\mathbf{I}$. We also assume $\mathbf{n}_{1}$ and $\mathbf{n}_{2}$ have same statistics as $\mathbf{n}$ in (1). Denote by $\mathbf{K}_{\ell, 0}$ and $\mathbf{D}_{\ell, 0}$ the FFE and FBE at the $\ell$ th iteration for $\mathrm{CH} 1$, and let $\mathbf{A}_{\ell, 0}=\operatorname{diag}\left(\mathbf{K}_{\ell, 0}^{\mathrm{H}} \mathbf{H}_{0}\right)$. Under the assumptions AS2)-AS4), we have the following results.

1) The FFE $\mathbf{K}_{\ell}$ and FBE $\mathbf{D}_{\ell}$ of the MIMO channel $\mathrm{CH} 2$ can be represented as

$$
\begin{aligned}
& \mathbf{K}_{\ell}=\mathbf{K}_{\ell, 0} \mathbf{E}^{\mathrm{H}} \\
& \mathbf{D}_{\ell}=\mathbf{E D}_{\ell, 0} \mathbf{E}^{\mathrm{H}}+\rho_{\ell-1}\left(\operatorname{diag}\left(\mathbf{E} \mathbf{K}_{\ell, 0}^{\mathrm{H}} \mathbf{H}_{0} \mathbf{E}^{\mathrm{H}}\right)-\mathbf{E} \mathbf{A}_{\ell, 0} \mathbf{E}^{\mathrm{H}}\right)
\end{aligned}
$$

2) If $\mathbf{H}_{0}$ is a diagonal matrix, and the elements of $\mathbf{E}$ are all with the same amplitude, then $\mathbf{K}_{\ell, 0}$ and $\mathbf{A}_{\ell, 0}=\mathbf{K}_{\ell, 0}^{\mathrm{H}} \mathbf{H}_{0}$ are diagonal matrices, and

$$
\begin{aligned}
\mathbf{K}_{\ell}^{\mathrm{H}} \mathbf{H} & =\mathbf{E A}_{\ell, 0} \mathbf{E}^{\mathrm{H}}, \quad \mathbf{A}_{\ell}=\operatorname{diag}\left(\mathbf{K}_{\ell}^{\mathrm{H}}\right)=\bar{a}_{\ell, 0} \mathbf{I} \\
\mathbf{D}_{\ell} & =\rho_{\ell-1} \mathbf{E}\left(\bar{a}_{\ell, 0} \mathbf{I}-\mathbf{A}_{\ell, 0}\right) \mathbf{E}^{\mathrm{H}} \\
\mathbf{R}_{\tilde{n}_{\ell}}(k, k) & =\left(\frac{1}{P}\right) \operatorname{Tr}\left(\mathbf{R}_{\ell, 0}\right)
\end{aligned}
$$

where $\bar{a}_{\ell, 0}=(1 / P) \operatorname{Tr}\left(\mathbf{A}_{\ell, 0}\right)$, and $\mathbf{R}_{\ell, 0}=\sigma_{s}^{2}(1-$ $\left.\rho_{\ell-1}^{2}\right)\left(\mathbf{A}_{\ell, 0}-\bar{a}_{\ell, 0} \mathbf{I}\right)\left(\mathbf{A}_{\ell, 0}-\bar{a}_{\ell, 0} \mathbf{I}\right)^{\mathrm{H}}+\sigma_{n}^{2} \mathbf{K}_{\ell, 0}^{\mathrm{H}} \mathbf{K}_{\ell, 0}$ is a diagonal matrix.

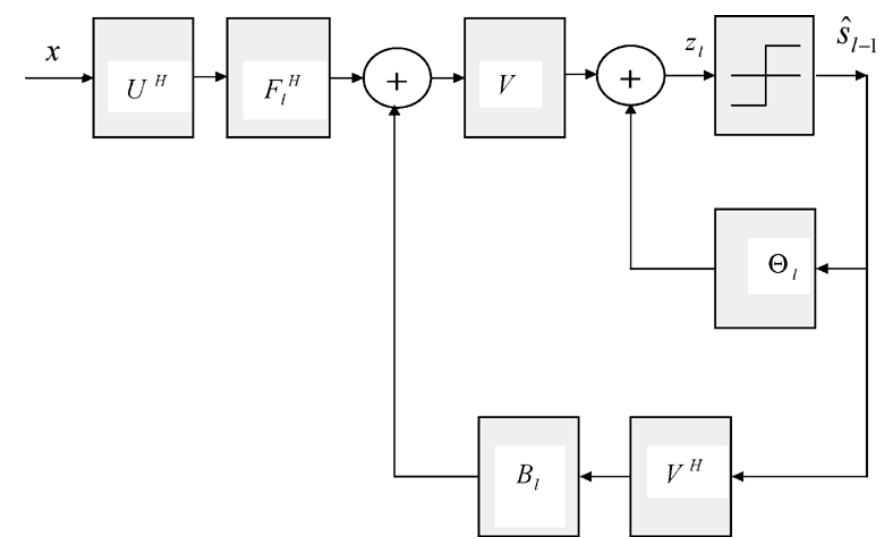

Fig. 2. Block diagram of SVD-based BI-GDFE receiver for generic MIMO systems.

The proof of Theorem 2 is straightforward. According to Theorem 1, for $\mathrm{CH} 1$, we have

$$
\begin{aligned}
& \mathbf{K}_{\ell, 0}=\left[\left(1-\rho_{\ell-1}^{2} \mathbf{H}_{0} \mathbf{H}_{0}^{\mathrm{H}}+\left(\frac{1}{\gamma}\right) \mathbf{I}\right]^{-1} \mathbf{H}_{0}\right. \\
& \mathbf{D}_{\ell, 0}=\rho_{\ell-1}\left(\mathbf{A}_{\ell, 0}-\mathbf{K}_{\ell, 0}^{\mathrm{H}} \mathbf{H}_{0}\right) .
\end{aligned}
$$

The FFE $\mathbf{K}_{\ell}$ and FBE $\mathbf{D}_{\ell}$ of $\mathrm{CH} 2$ shown in Part 1) can be obtained using (14) and (15), by inserting $\mathbf{H}=\mathbf{H}_{0} \mathbf{E}^{\mathrm{H}}$. The results of Part 2) can be derived as well.

With Theorem 2, we look at an equivalent version of Algorithm 1 using singular value decomposition (SVD). Decompose the channel matrix $\mathbf{H}$ using SVD: $\mathbf{H}=\mathbf{U} \boldsymbol{\Sigma} \mathbf{V}^{\mathrm{H}}$, where $\mathbf{U}$ is the $N \times N$ matrix of the left singular vectors of $\mathbf{H}, \mathbf{V}$ is the $P \times P$ matrix of the right singular vectors of $\mathbf{H}$, and $\boldsymbol{\Sigma}$ is the $N \times P$ singular value matrix of $\mathbf{H}(\boldsymbol{\Sigma}(i, i)$, $i=1, \ldots, \min (P, N)$, are the singular values). According to Theorem 2, the FFE of (14) can be written as: $\mathbf{K}_{\ell}=\mathbf{U} \mathbf{F}_{\ell} \mathbf{V}^{\mathrm{H}}$, where $\mathbf{F}_{\ell}=\left[\left(1-\rho_{\ell-1}^{2}\right) \boldsymbol{\Sigma} \boldsymbol{\Sigma}^{\mathrm{H}}+(1 / \gamma) \mathbf{I}_{N \times N}\right]^{-1} \boldsymbol{\Sigma}$. Similarly, the FBE of (15) can be written as

$$
\mathbf{D}_{\ell}=\rho_{\ell-1}\left(\mathbf{A}_{\ell}-\mathbf{K}_{\ell}^{\mathrm{H}} \mathbf{H}\right)=\rho_{\ell-1}\left(\bar{a}_{\ell} \mathbf{I}-\mathbf{K}_{\ell}^{\mathrm{H}} \mathbf{H}\right)+\rho_{\ell-1}\left(\mathbf{A}_{\ell}-\bar{a}_{\ell} \mathbf{I}\right)
$$

where $\bar{a}_{\ell}=(1 / P) \operatorname{Tr}\left(\mathbf{K}_{\ell}^{\mathrm{H}} \mathbf{H}\right)$. Substituting $\mathbf{K}_{\ell}=\mathbf{U F}_{\ell} \mathbf{V}^{\mathrm{H}}$ into (26) and using the SVD of $\mathbf{H}$ yield $\mathbf{D}_{\ell}=\mathbf{V B}_{\ell} \mathbf{V}^{\mathrm{H}}+\boldsymbol{\Theta}_{\ell}$, where $\mathbf{B}_{\ell}=\rho_{\ell-1}\left(\bar{a}_{\ell} \mathbf{I}-\mathbf{F}_{\ell}^{\mathrm{H}} \boldsymbol{\Sigma}\right)$ and $\boldsymbol{\Theta}_{\ell}=\rho_{\ell-1}\left(\mathbf{A}_{\ell}-\bar{a}_{\ell} \mathbf{I}\right)$. Therefore, the input signal to the slicer is given by

$$
\begin{aligned}
\mathbf{z}_{\ell} & =\mathbf{V} \mathbf{F}_{\ell}^{\mathrm{H}} \mathbf{U}^{\mathrm{H}} \mathbf{x}+\left(\mathbf{V} \mathbf{B}_{\ell} \mathbf{V}^{\mathrm{H}}+\boldsymbol{\Theta}_{\ell}\right) \hat{\mathbf{s}}_{\ell-1} \\
& =\mathbf{V}\left(\mathbf{F}_{\ell}^{\mathrm{H}} \mathbf{U}^{\mathrm{H}} \mathbf{x}+\mathbf{B}_{\ell} \mathbf{V}^{\mathrm{H}} \hat{\mathbf{s}}_{\ell-1}\right)+\boldsymbol{\Theta}_{\ell} \hat{\mathbf{s}}_{\ell-1} .
\end{aligned}
$$

From these derivations, the block diagram of the equivalent receiver is shown in Fig. 2. The receiver algorithm based on the above derivation is referred to as Algorithm 2.

Finally, a simplified BI-GDFE is illustrated in Fig. 3. Compared to Fig. 2, there is no $\boldsymbol{\Theta}_{\ell}$ branch in Fig. 3. The performance comparison for different BI-GDFEs shown in Figs. 2 and 3 will be given in Section VII.

\section{IDC SELECTION}

The convergence of the proposed iterative receiver is very sensitive to the selection of the IDC coefficient $\rho_{\ell-1}$. If the 


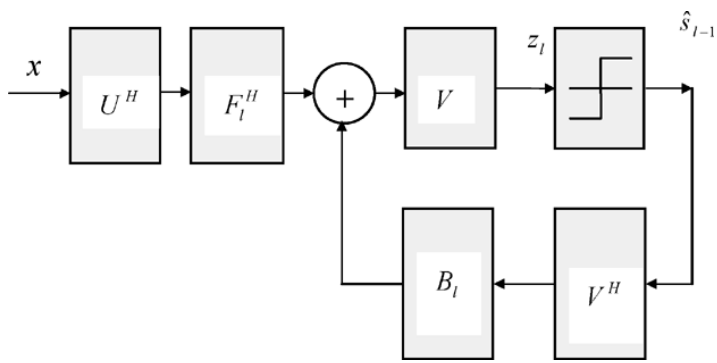

Fig. 3. Block diagram of simplified BI-GDFE receiver for generic MIMO systems.

signal dimension $P$ is infinity, the IDC coefficient can be calculated based on the output SINR using Gaussian approximation [5], [6]. In practice, the signal dimension is usually finite and possibly small, thus we have to design our receiver by replacing $\rho_{\ell-1}$ with $(1 / P) \sum_{k} s(k) \hat{s}_{\ell-1}^{*}(k)$, which is the arithmetic mean of $s(k) \hat{s}_{\ell-1}^{*}(k)$. Unfortunately, this arithmetic mean is unavailable since the transmitted symbols are not known. Further, no matter the equalized SINRs for different detected symbols are identical or not, the IDC $\rho_{\ell-1}$ estimated through arithmetic mean will fluctuate from one block of $P$ transmitted symbols to another block, since the realizations of the noise are different, and in fact, this fluctuation increases when the signal dimension $P$ decreases. Therefore, it is desirable to design the IDC such that the proposed BI-GDFE receiver can work for systems with finite signal dimension and unequal uqualized SINRs.

Let us look at the noise-plus-interference covariance (NIC) matrix (47) given in Appendix $B$, and choose $\mathbf{D}_{\ell}=-\hat{\rho}_{\ell-1} \mathbf{C}_{\ell}$, where $\hat{\rho}_{\ell-1}$ is the selected IDC, which may be different from the true IDC $\rho_{\ell-1}$ calculated with arithmetic mean. Because of the mismatch, the resultant NIC matrix is given by

$$
\begin{aligned}
\tilde{\mathbf{R}}_{\tilde{n}_{\ell}=} & \sigma_{s}^{2}\left(1-\rho_{\ell-1}^{2}\right) \mathbf{C}_{\ell} \mathbf{C}_{\ell}^{\mathrm{H}}+\sigma_{s}^{2}\left(\rho_{\ell-1}-\hat{\rho}_{\ell-1}\right)^{2} \mathbf{C}_{\ell} \mathbf{C}_{\ell}^{\mathrm{H}} \\
& +\sigma_{n}^{2} \mathbf{K}_{\ell}^{\mathrm{H}} \mathbf{K}_{\ell} \\
= & \sigma_{s}^{2}\left(1-\hat{\rho}_{\ell-1}^{2}\right) \mathbf{C}_{\ell} \mathbf{C}_{\ell}^{\mathrm{H}}+2 \hat{\rho}_{\ell-1}\left(\hat{\rho}_{\ell-1}-\rho_{\ell-1}\right) \sigma_{s}^{2} \mathbf{C}_{\ell} \mathbf{C}_{\ell}^{\mathrm{H}} \\
& +\sigma_{n}^{2} \mathbf{K}_{\ell}^{\mathrm{H}} \mathbf{K}_{\ell} .
\end{aligned}
$$

It is seen that if the selected IDC $\hat{\rho}_{\ell-1}$ is not equal to the true IDC, $\rho_{\ell-1}$, compared to (47) in Appendix B, there exists an additional positive term, $\sigma_{s}^{2}\left(\rho_{\ell-1}-\hat{\rho}_{\ell-1}\right)^{2} \mathbf{C}_{\ell} \mathbf{C}_{\ell}^{\mathrm{H}}$, which contributes to decreased SINR for the future iterations.

When $\hat{\rho}_{\ell-1}$ is selected, even $\hat{\rho}_{\ell-1}$ may not be equal to the real IDC $\rho_{\ell-1}$ exactly, we still have to calculate the NIC matrix as follows:

$$
\hat{\mathbf{R}}_{\tilde{n}_{\ell}}=\sigma_{s}^{2}\left(1-\hat{\rho}_{\ell-1}^{2}\right) \mathbf{C}_{\ell} \mathbf{C}_{\ell}^{\mathrm{H}}+\sigma_{n}^{2} \mathbf{K}_{\ell}^{\mathrm{H}} \mathbf{K}_{\ell} .
$$

The above will further be used for SINR calculation. Now, we look at the impact of the IDC mismatch on the convergence of the proposed algorithm.

1) If the IDC is over-estimated, i.e., $\hat{\rho}_{\ell-1}>\rho_{\ell-1}$, then $\tilde{\mathbf{R}}_{\tilde{n}_{\ell}}>\hat{\mathbf{R}}_{\tilde{n}_{\ell}}$. It is treated that most of the interferences have been cancelled out even though they are actually not, thus a closer-to-SUMF weight matrix is designed for the FFE. Convergence may not be guaranteed in this case.
2) If the IDC is under-estimated, i.e., $\hat{\rho}_{\ell-1}<\rho_{\ell-1}$, then $\tilde{\mathbf{R}}_{\tilde{n}_{\ell}}<\hat{\mathbf{R}}_{\tilde{n}_{\ell}}$. A lower-than-expected SINR is utilized for generating IDC and FFE and FBE weights for the next iteration. While this may generally lead to a converged solution, the convergence speed is usually very slow, and thus higher receiver complexity will be required due to the increased number of iterations.

Therefore, it is critical to choose the estimated IDC $\hat{\rho}_{\ell-1}$ as close to the true IDC $\rho_{\ell-1}$ as possible so that fast and guaranteed convergence can be achieved. As aforementioned, for finite signal dimensions, the true IDCs are unknown, and they are dependent on the signal dimension as well as the SINR of the equalization output. Here we propose an algorithm to determine the IDCs with which the proposed BI-GDFE can work even for limited block size. Using central limit theorem, the interference and noise after equalization can be treated as a complex Gaussian variable, thus the equalization output at each iteration can be modeled as the output of an AWGN channel whose SNR being the SINR of equalization output at that iteration. Our idea is to design one IDC for each pair of signal dimension $P$, and "average" output SINR, $\Gamma$. The IDC determination algorithm consists of the following steps.

Step 1:For an AWGN channel with SNR $\Gamma$, generate a block of $P$ modulated symbols, and make hard decisions over the received signals, then calculate the cross-correlation between the transmitted signals with the decision symbols. Repeat this process for $S$ runs, say $S=10000$, from which we obtain 10000 realizations of IDCs for the SNR/signal dimension pair $(\Gamma, P)$. Step 2:Calculate the mean, $\bar{\rho}(\Gamma, P)$, of these IDC coefficients, which should be close to (8) for QPSK modulation;

Step 3:Search the minimum IDC coefficient, $\rho_{\min }(\Gamma, P)$, from the $S$ realizations. This minimum is actually the worst case IDC. Step 4:Calculating the average between the mean IDC and minimum IDC, yields $\rho(\Gamma, P)=(1 / 2)\left(\bar{\rho}(\Gamma, P)+\rho_{\min }(\Gamma, P)\right)$, which serves as the IDC value for the $(\Gamma, P)$ pair. This value will be used in BI-GDFE when the signal dimension is $P$, and output average SINR is $\Gamma$.

Step 5:Vary the $(\Gamma, P)$ pair to obtain the IDC versus SNR/signal dimension curves.

Selecting the IDC as the the average of the minimum and the mean of the simulated correlations, makes a tradeoff between convergence speed and guarantee of convergence. A lookup table can be generated based on the measured curves, and when the systems have identical equalized SINRs, the checked value can be applied directly. For systems with unequal equalized SINRs, two procedures can be applied. One is to use the geometric mean of the SINRs of the detected symbols. In this case, the calculation of geometric mean can be very expensive. The 
TABLE I

COMPLEXITY COMPARISON FOR ALGORITHM 1 AND ALGORITHM 2

\begin{tabular}{||l|c|c|c|c||}
\hline \hline Algorithms & Complex Multiplications (CMs) & $L=4$ & $L=6$ & $L=30$ \\
\hline Algorithm 1 & $L\left(\mu_{1}+1\right) P^{3}+2 L Q P^{2}$ & $8.8 \times 10^{6}$ & $1.3 \times 10^{6}$ & $6.6 \times 10^{7}$ \\
\hline Algorithm 2 & $\left(\mu_{2}+L\right) P^{3}+2 L Q P^{2}+3 L Q P$ & $1.0 \times 10^{7}$ & $1.3 \times 10^{6}$ & $4.2 \times 10^{7}$ \\
\hline \hline
\end{tabular}

other solution is to use the average SINR, defined as the ratio between the average signal power to the average interference plus noise power. This method simplifies the calculations significantly.

\section{Suboptimal BI-GDFE}

The complexity of the BI-GDFE receiver includes two parts: pre-processing complexity for calculating the weights, and on-line processing complexity for recovering the $P$ symbols. For block fading channels, the equalizer weights can be utilized for the whole packet of say $Q$ blocks, each requiring separate iterations for on-line processing. Here, we compare the complexity of Algorithm 1 and Algorithm 2 assuming $P=N$.

For Algorithm 1, the pre-processing for each iteration consists of the calculation of $\mathbf{K}_{\ell}, \mathbf{D}_{\ell}$ and $\gamma_{\ell}$. Specifically, the computation of $\mathbf{K}_{\ell}$ requires $\mu_{1} P^{3}$ complex multiplications (CMs), where $\mu_{1}$ is a scaling factor depending on the algorithm used for matrix inversion. The computation of $\mathbf{D}_{\ell}$ and $\gamma_{\ell}$ requires $P^{3} \mathrm{CMs}$. For on-line processing, each iteration consists of $2 P^{2}$ CMs. So, if $L$ iterations are required, the number of required CMs for one packet of $Q$ blocks is $L\left(\mu_{1}+1\right) P^{3}+2 L Q P^{2}$.

For Algorithm 2, the preprocessing consists of one time SVD calculation for channel matrix $\mathbf{H}$, which requires $\mu_{2} P^{3} \mathrm{CMs}$. The calculation of $\mathbf{F}_{\ell}, \mathbf{B}_{\ell}, \boldsymbol{\Theta}_{\ell}$ and $\boldsymbol{\gamma}_{\ell}$ involves with $P^{3} \mathrm{CMs}$ (mainly due to the calculation of SINR $\gamma_{\ell}$ ). For on-line processing, each iteration involves $2 P^{2}+3 P$ CMs. So, if $L$ iterations are required, the number of required $\mathrm{CMs}$ for one packet of $Q$ blocks is $\mu_{2} P^{3}+L P^{3}+2 L Q P^{2}+3 L Q P$.

Table I shows the complexity comparison for the two algorithms for different number $L$ of required iterations with $Q=$ $10, P=100$, and assuming $\mu_{1}=1$ and $\mu_{2}=6$. It is seen that if the number of required iterations $L$ is small, then Algorithm 1 is simpler than Algorithm 2; however, if the number of required iterations is large, Algorithm 2 is more computationally efficient.

The calculation of matrix inversion or SVD can be simplified using say reduced rank approximation methods [21], [22]. In both algorithms, it is noted that the calculation of equalized SINR $\gamma_{\ell}$ occupies a very heavy complexity load, especially when the number $L$ of iterations is large. In order to simplify the calculations, for Algorithm 2, we approximate the SINR for the $\ell$ th iteration as follows:

$$
\gamma_{\ell}=\frac{\bar{a}_{\ell}^{2}}{\left(\frac{1}{N}\right) \operatorname{Tr}\left(\left(1-\rho_{\ell-1}^{2}\right) \mathbf{B}_{\ell} \mathbf{B}_{\ell}^{\mathrm{H}}+\left(\frac{1}{\gamma}\right) \mathbf{F}_{\ell}^{\mathrm{H}} \mathbf{F}_{\ell}\right)} .
$$

Since both $\mathbf{F}_{\ell}$ and $\mathbf{B}_{\ell}$ are diagonal matrices, the calculation of $\gamma_{\ell}$ requires only $2 N$ CMs.

To alleviate the requirement of calculating the SINR and of determining the IDC, we further propose a fixed IDC selection method, which chooses a fixed set of IDCs for the BI-GDFE algorithm. By using iterations, the decisions are getting more and more reliable, thus an increasing IDC with respect to the iteration numbers will be selected, and the upper bound of the IDC is 1 . The performance of this fixed IDC selection will also be evaluated in the simulations.

\section{Asymptotic PeRformance ANAlysis}

In this section, we study the asymptotic performance of the proposed BI-GDFE receiver for random MIMO channels, assuming that $P \rightarrow \infty, N \rightarrow \infty$, and $P / N \rightarrow \alpha$, where $\alpha$ is a fixed constant, and can be greater than one. First, let us introduce the asymptotic results for the SINR of the conventional MMSE receiver. For that, we modify the assumptions AS1) and AS2) as follows.

AS1m) In AS1), we further assume that $h_{i, k} \mathrm{~s}$ are iid for all $i$ s and $k \mathrm{~s}$.

AS2m) The received power for user $i$ is denoted as $p_{i}$.

Note we add the iid assumption to the channel coefficients in AS1m), but relax the received powers to be possibly unequal in AS2m). With the assumptions AS1m), AS2m), and AS3), the MMSE weight for recovering user 1 is given by

$$
\mathbf{k}_{1}=\left(\mathbf{H}_{1} \mathbf{G}_{1} \mathbf{H}_{1}^{\mathrm{H}}+\left(\frac{1}{\gamma}\right) \mathbf{I}\right)^{-1} \mathbf{h}_{1}
$$

where $\mathbf{G}_{1}=\operatorname{diag}\left(p_{2}, \ldots, p_{P}\right)$. Please note $p$ denotes the power, the subscript ${ }_{P}$ here is the signal dimension, or number of users. The SINR of the MMSE receiver for recovering user 1 is represented as

$$
\bar{\beta}^{(N)}=p_{1} \mathbf{h}_{1}^{\mathrm{H}}\left(\mathbf{H}_{1} \mathbf{G}_{1} \mathbf{H}_{1}^{\mathrm{H}}+\left(\frac{1}{\gamma}\right) \mathbf{I}\right)^{-1} \mathbf{h}_{1} .
$$

While the MMSE weights are dependent on the (random) channel coefficients, according to [11], [12], when $P \rightarrow \infty$, $N \rightarrow \infty$, and $P / N \rightarrow \alpha$, the SINR of the MMSE equalization output converges to a fixed value, which depends on the limiting power distribution of the interferers only.

Proposition 1 [11]: Consider the MIMO channel (1). Under assumptions AS1m), AS2m), and AS3), and suppose the empirical distribution function of $\mathbf{G}_{1}$ converges to a probability distribution function $F_{p}(x)$, then the SINR $\bar{\beta}^{(N)}$ converges to $\bar{\beta}^{*}$ in probability as $P \rightarrow \infty, N \rightarrow \infty$, and $P / N \rightarrow \alpha$, where $\bar{\beta}^{*}$ is the positive solution (which is unique) to

$$
\bar{\beta}^{*}=\frac{P_{1}}{\sigma_{n}^{2}+\alpha \int \frac{p p_{1}}{p_{1}+p \bar{\beta}^{*}} d F_{p}(x)} .
$$


In general, there is no explicit solution for the SINR $\bar{\beta}^{*}$ in (34). However, when all users (data streams) have same received SNR, $\gamma$, the limiting SINR has a simple expression, which is described by the following proposition [11], [12].

Proposition 2: Consider the MIMO channel model (1). Under assumptions AS1m), AS2m), and AS3), the SINR $\beta^{(N)}(\gamma)=\mathbf{h}_{1}^{\mathrm{H}}\left(\mathbf{H}_{1} \mathbf{H}_{1}^{\mathrm{H}}+(1 / \gamma) \mathbf{I}\right)^{-1} \mathbf{h}_{1}$ of the MMSE receiver converges to $\beta^{*}(\gamma)$ in probability as $P \rightarrow \infty, N \rightarrow \infty$, and $P / N \rightarrow \alpha$, where $\beta^{*}(\gamma)$ is given by

$$
\beta^{*}(\gamma)=\frac{(1-\alpha) \gamma}{2}-\frac{1}{2}+\sqrt{\frac{(1-\alpha)^{2} \gamma^{2}}{4}+\frac{(1+\alpha) \gamma}{2}+\frac{1}{4}} .
$$

For the limiting case, we have proved that AS4) is almost surely true. Thus, the solutions derived in Theorem 1 are exact. We are now ready to introduce the main results for the limiting SINRs of the proposed BI-GDFE receiver when all users are with equal power.

Theorem 3: Consider the MIMO channel model (1). Under the assumptions AS1m), AS2m), and AS3), the SINR $\beta_{\ell}^{(N)}(\gamma)$ of any user using BI-GDFE receiver at the $\ell$ th iteration converges to $\beta_{\ell}^{*}(\gamma)$ in probability as $P \rightarrow \infty, N \rightarrow \infty$, and $P / N \rightarrow \alpha$, where $\beta_{\ell}^{*}(\gamma)$ is given by

$$
\begin{aligned}
& \beta_{\ell}^{*}(\gamma)=\frac{1}{1-\rho_{\ell-1}^{2}}\left[\frac{(1-\alpha)\left(1-\rho_{\ell-1}^{2}\right) \gamma}{2}-\frac{1}{2}\right. \\
& \left.+\sqrt{\frac{(1-\alpha)^{2}\left(1-\rho_{\ell-1}^{2}\right)^{2} \gamma^{2}}{4}+\frac{(1+\alpha)\left(1-\rho_{\ell-1}^{2}\right) \gamma}{2}+\frac{1}{4}}\right]
\end{aligned}
$$

for $\rho_{\ell-1}<1$, and $\beta_{\ell}^{*}(\gamma)=\gamma$ for $\rho_{\ell-1}=1$.

Proof: According to (48), the output SINR of user $i$ using BI-GDFE receiver at the $\ell$ th iteration is

$$
\begin{aligned}
\beta_{\ell}^{(N)}(\gamma) & =\frac{\left|\mathbf{k}_{i}^{\mathrm{H}} \mathbf{h}_{i}\right|^{2}}{\left(1-\rho_{\ell-1}^{2}\right) \sum_{j \neq i}\left|\mathbf{k}_{i}^{\mathrm{H}} \mathbf{h}_{j}\right|^{2}+\left(\frac{1}{\gamma}\right)\left\|\mathbf{k}_{i}\right\|^{2}} \\
& =\frac{1}{\left(1-\rho_{\ell-1}^{2}\right)} \frac{\left|\mathbf{k}_{i}^{\mathrm{H}} \mathbf{h}_{i}\right|^{2}}{\sum_{j \neq i}\left|\mathbf{k}_{i}^{\mathrm{H}} \mathbf{h}_{j}\right|^{2}+\frac{1}{\gamma\left(1-\rho_{\ell-1}^{2}\right)}\left\|\mathbf{k}_{i}\right\|^{2}} .
\end{aligned}
$$

The second multiplication component of the right-hand side (RHS) of (38) is the output SINR of the conventional MMSE receiver for channel model (1) with receive SNR $\bar{\gamma}=\gamma\left(1-\rho_{\ell-1}^{2}\right)$, or we can write $\beta_{\ell}^{(N)}(\gamma)=\left(1 /\left(1-\rho_{\ell-1}^{2}\right)\right) \beta^{(N)}(\bar{\gamma})$. Thus as $P \rightarrow \infty, N \rightarrow \infty$, and $P / N \rightarrow \alpha, \beta_{\ell}^{(N)}(\gamma)$ converges to

$$
\beta_{\ell}^{*}(\gamma)=\frac{1}{\left(1-\rho_{\ell-1}^{2}\right)} \beta^{*}(\bar{\gamma}) .
$$

Equation (36) can be obtained by inserting (35) into (39).

Theorem 3 tells us that the limiting SINRs for all users/data streams are equal. Thus, the statistical reliability $\rho_{\ell}$ can be computed exactly, using (8) for QPSK modulation. The updates from $\beta_{\ell-1}$ to $\rho_{\ell-1}$, and then from $\rho_{\ell-1}$ to $\beta_{\ell}$ will push $\beta_{\ell}$ to increase gradually. The upper-bound $\gamma$ can be reached when the IDC becomes 1 . This means that the received SNR will serve as the upper-bound for the SINR of the BI-GDFE receiver, and if this

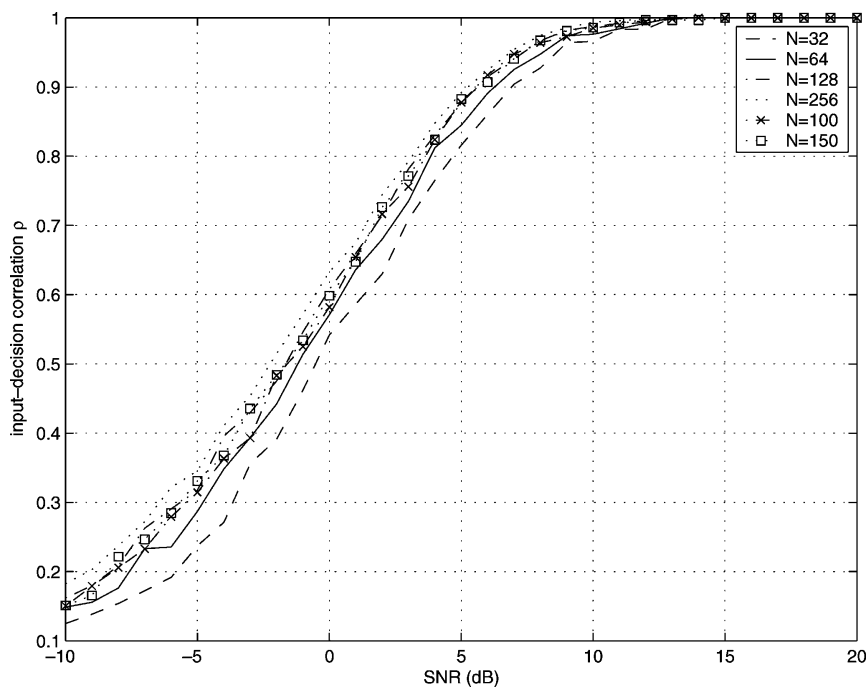

Fig. 4. IDC curves for different SNRs and signal dimensions.

bound is achievable, all transmitted symbols are completely decoupled without any loss.

\section{COMPUTER Simulations}

We compare the proposed iterative receiver with the conventional MMSE receiver, which also provides the initial FFE for the proposed receiver. Due to the large signal dimensions, we are unable to provide the ML bound for those systems. Thus, the single user MFB is used to serve as the lower-bound for assessing the effectiveness of the proposed receiver. The single user MFB is derived assuming that the interferences have been completely cancelled out and the desired signals have been coherently combined.

The IDC coefficients are first shown in Fig. 4 for different block sizes and input SNRs. These curves are derived using the method presented in Section IV with 2000 Monte Carlo runs for each pair of SNR and block size. It is noted that with the increase of the block size, the equalized SINRs and thus the IDCs become less fluctuated, thus, a larger IDC coefficient can be applied in designing the FBE and FFE as compared to the case with a smaller block size. For the limiting case, the IDC is exactly derived using (8).

\section{A. Random MIMO Systems}

We first simulate the large random MIMO systems with $P=N=100$. Each element of the MIMO channel follows zero mean, unit variance circularly symmetric complex Gaussian (CSCG) distribution. This situation represents frequency-flat synchronous CDMA uplink with random spreading codes, or MIMO antenna systems with large antenna sizes. While the latter looks like not so realistic, if we really can build a $100 \times 100$ MIMO antenna systems, the uncoded data rate can be as high as $4 \mathrm{Gbits} / \mathrm{s}$ when a bandwidth of $20 \mathrm{MHz}$ is utilized.

Fixed IDC Selection: The IDC coefficients are selected as follows: $\left[\rho_{1}, \ldots, \rho_{4}\right]=[0.95,0.95,0.99,0.999]$. This IDC 


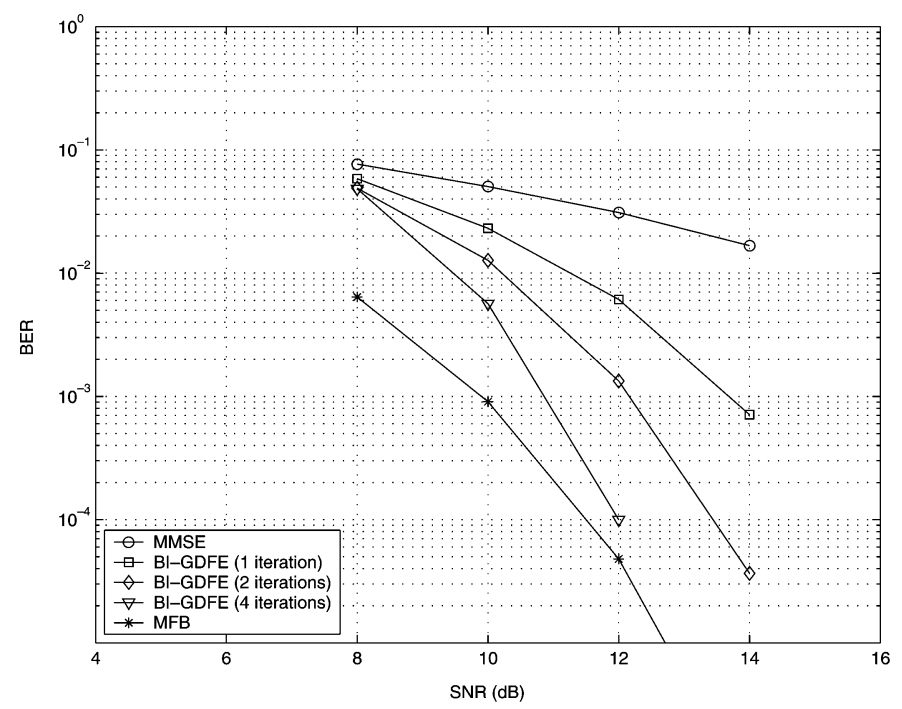

(a)

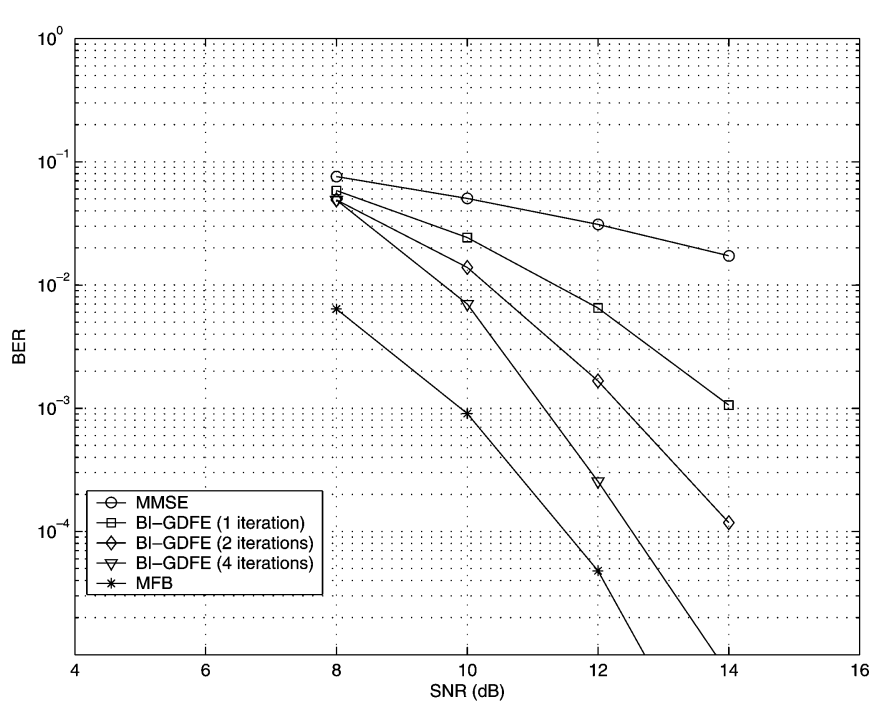

(b)

Fig. 5. Performance of random MIMO systems with fixed IDC. (a) $100 \times 100$, BI-GDFE. (b) $100 \times 100$, suboptimal BI-GDFE.

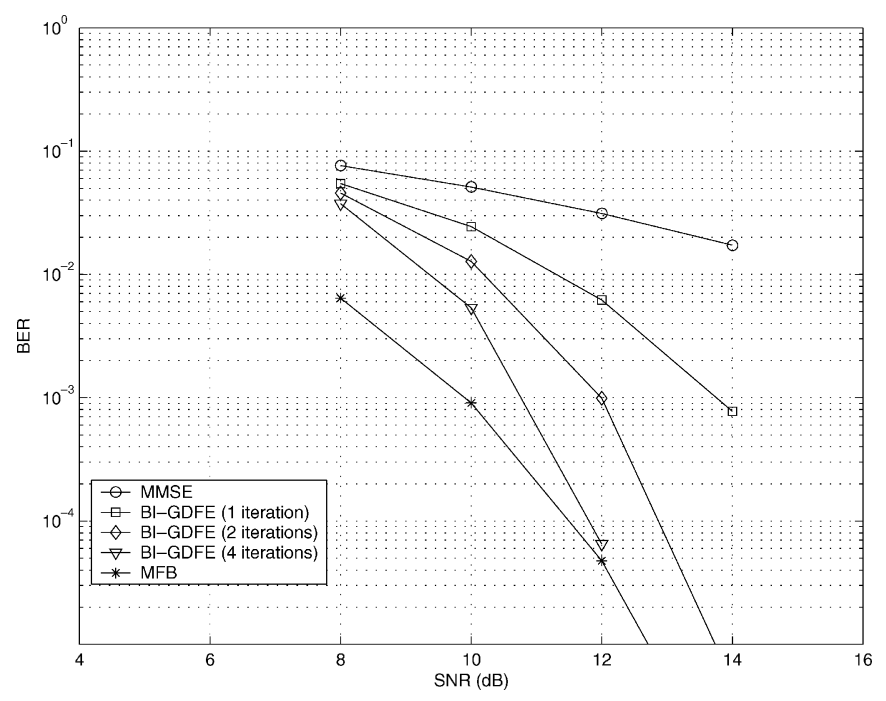

Fig. 6. Performance of BI-GDFE for $100 \times 100$ random MIMO systems with dynamic IDC selection.

selection simplifies the calculation for SINRs. Fig. 5 shows the BER versus SNR results for the two MIMO systems using BI-GDFE with $\Theta_{\ell}$ branch (see Fig. 2), and supoptimal BI-GDFE without $\boldsymbol{\Theta}_{\ell}$ branch (see Fig. 3). It is seen that the proposed scheme achieves dramatic performance improvement over the conventional MMSE receiver, and there is a slight performance loss for the supoptimal BI-GDFE.

Dynamic IDC Selection: Fig. 6 shows the BER versus SNR results for $100 \times 100 \mathrm{MIMO}$ antenna system using dynamic IDC selection based on the IDC curves shown in Fig. 4 where the input SNR is the equalized SINR calculated with (48). It is seen that the proposed method almost achieves the MFB using only four iterations when the SNR is above $12 \mathrm{~dB}$.
We point out that the fixed IDC values are selected based on a try-and-compare basis, and they are almost the best we can have so far for QPSK modulation. However, in the low SNR region, these values seem to be not so good since the BER for the fourth iteration is worse than that for the third iteration when the SNR is lower than $8 \mathrm{~dB}$. In other words, this set of values may be close to the optimum for SNR values around $12 \mathrm{~dB}$, but may not be good for low SNRs. However, using dynamic IDC selection, we can generally achieve decreased BERs with the iterations, and the price we have to pay is the increased complexity to calculate the SINRs for each iterations, and to find out the IDCs for the calculated SINRs.

To further evaluate the importance of using dynamic IDC selection for small block size, Fig. 7 compares the dynamic IDC method and the IDC method of [6], which is based on (8), for $32 \times 32$ antenna systems. It is seen that if the theoretical values designed for limiting case are utilized, the achievable performance after six iterations is still far from the MFB. However, the proposed IDC selection method makes the achievable performance just about $2 \mathrm{~dB}$ away the MFB for the SNR region we are concerned here. Thus the superiority of the new method over the method proposed in [6] is obvious. In this simulation, we have also simulated the MMSE-based VBLAST receiver, whose performance stays between the conventional MMSE receiver and one iteration BI-GDFE receiver.

\section{B. Asymptotic Performance}

We now look at the asymptotic performance of the proposed BI-GDFE receiver when applied to large random systems, when $P \rightarrow \infty, N \rightarrow \infty$ with $P / N \rightarrow \alpha$. Note the limiting traffic load $\alpha$ can be greater than 1 for random channels. Fig. 8 illustrates the asymptotic BER performance of the proposed BI-GDFE for each of the iterations with different traffic load $\alpha$ s. Here QPSK modulation is used, and the single user MFB is equal to the 


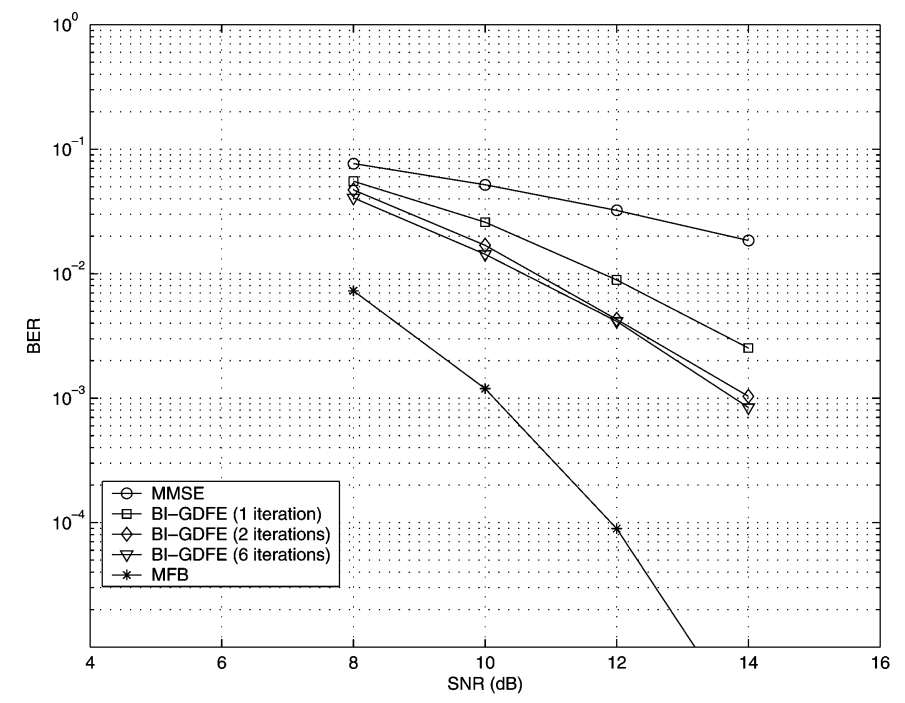

(a)

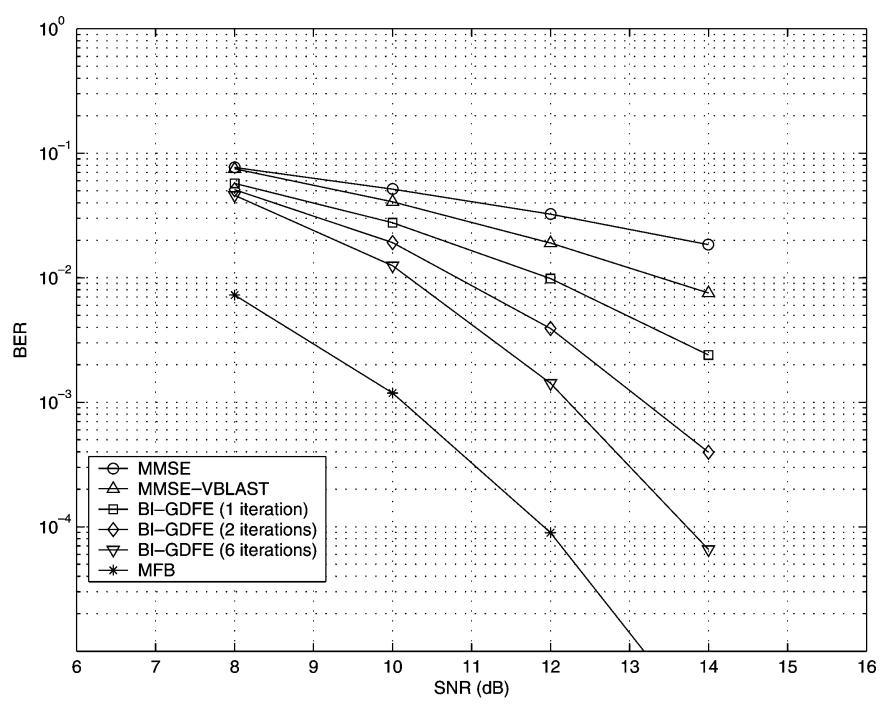

(b)

Fig. 7. Performance of $32 \times 32$ random MIMO systems using different IDC selection method. (a) Method proposed in [6]. (b) Method proposed in this paper. The results for MMSE-based VBLAST receiver are also included in (b).

single user AWGN bound (when $N \rightarrow \infty$, and all interferences are perfectly cancelled out, the SINR of the matched-filter output is the same as the received SNR, which provides the single user AWGN bound). In each figure, the upper-most curve is the BER-SNR curve for the conventional MMSE receiver. The lower-most curve is the BER-SNR curve for the single user AWGN bound. The other curves are the BER-SNR performance curves of the proposed BI-GDFE receiver for each of the iteration, with the increase of the iteration, the BER gets lower and lower but bounded by the AWGN bound.

It is seen that, for $\alpha \leq 1$, four iterations are sufficient for the BI-GDFE to converge to the AWGN bound when the received SNR $\gamma \geq 10 \mathrm{~dB}$. When $\alpha$ increases, more iterations are needed to make the algorithm converge. For $1 \leq \alpha \leq 2$, we still observe the convergence to the AWGN bound for $\gamma \geq 10 \mathrm{~dB}$. For $\alpha$ as high as 2.66, the convergence can still be observed, but the SNR region for the convergence is above $22 \mathrm{~dB}$. It is also seen that when $\alpha$ increases, the BI-GDFE receiver converges very slow for initial part of the iterations, but after a threshold number, it can converge to the bound very quickly.

Finally, to show the convergence of the output SINR to the received SNR, the dynamics of the SINRs of the proposed BI-GDFE are illustrated in Fig. 9 for $\alpha=1$. It is seen that the equalization output SINRs converge to the receive SNRs after 4 iterations when received SNR, $\gamma$, is greater than or equal to $10 \mathrm{~dB}$. Note the " $\rightarrow$ " shown in Fig. 9 denotes the direction of iteration increase.

\section{Random MIMO Systems With $P / N>1$}

In the asymptotic results, we have seen that the proposed receiver also works for the case $P / N>1$. Here, we presented the results for limited MIMO dimensions $P=100$ and $N=$ 90. As seen in Fig. 10, significant gain is achievable using the proposed BI-GDFE receiver as compared to the conventional MMSE receiver.

\section{MIMO-SCCP Systems for Frequency Selective Channels}

To evaluate the performance of the proposed receiver for frequency selective channels, we provide the results for MIMOSCCP systems. The MIMO system has two transmitters and two receivers, and the block size for SCCP is $N_{c}=64$. Fig. 11 shows the BER curves for the conventional MMSE receiver, the proposed BI-GDFE receiver with different iterations and the single user MFB. Here the results are derived assuming that the frequency domain channel responses are uncorrelated. However, similar results can be obtained even when they are correlated, which is the case when the multipath components are not so rich. Again, the convergence of the BI-GDFE receiver to the MFB is observed for an SNR of around $12 \mathrm{~dB}$.

\section{CONCLUSION}

In this paper, we have proposed a BI-GDFE receiver to iteratively and simultaneously (but not jointly) detect the transmitted symbols for large MIMO systems. By exploiting the input-decision correlation, a measure on the reliability of the earlier-made decisions, we design the feed-forward equalizers and feedback equalizers in such a way that maximized SINR is achieved in each iteration. Novel implementations are also proposed to simplify the receiver complexity drastically using singular value decomposition. The asymptotic performance of the proposed receiver is analyzed and evaluated through numerical results. Finally, computer simulations are presented to illustrate the effectiveness of the proposed receiver for MIMO channels with finite dimensions. It is illustrated that for random MIMO channels, the proposed BI-GDFE achieves the single user MFB within a few iterations. 


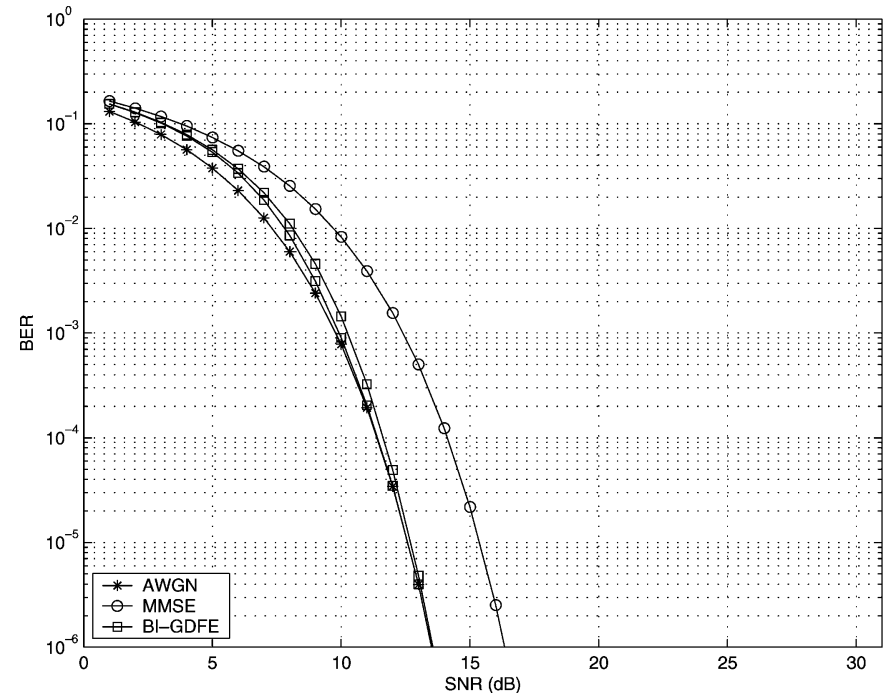

(a)

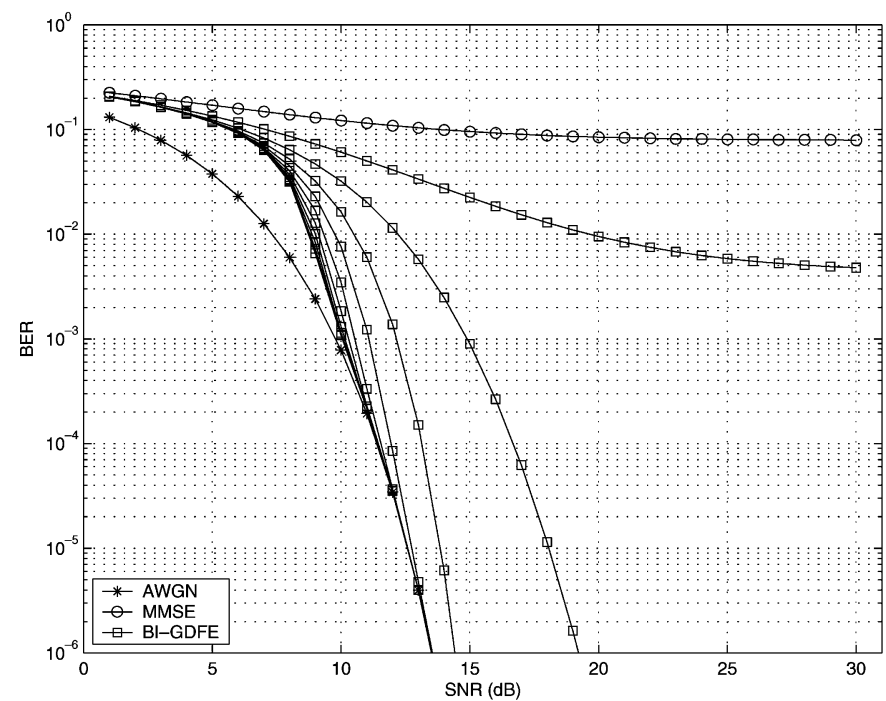

(c)

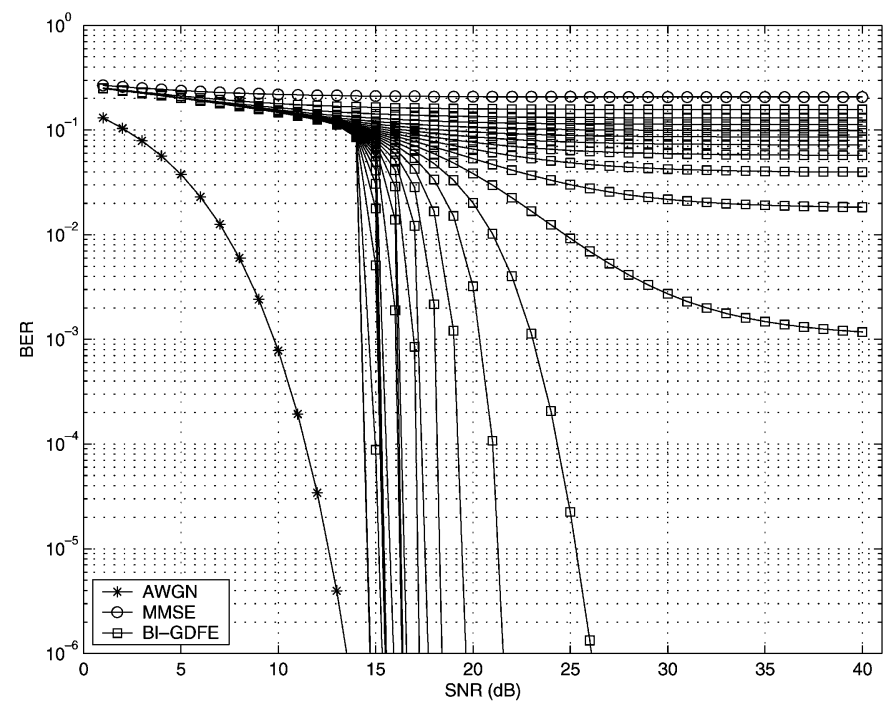

(e)

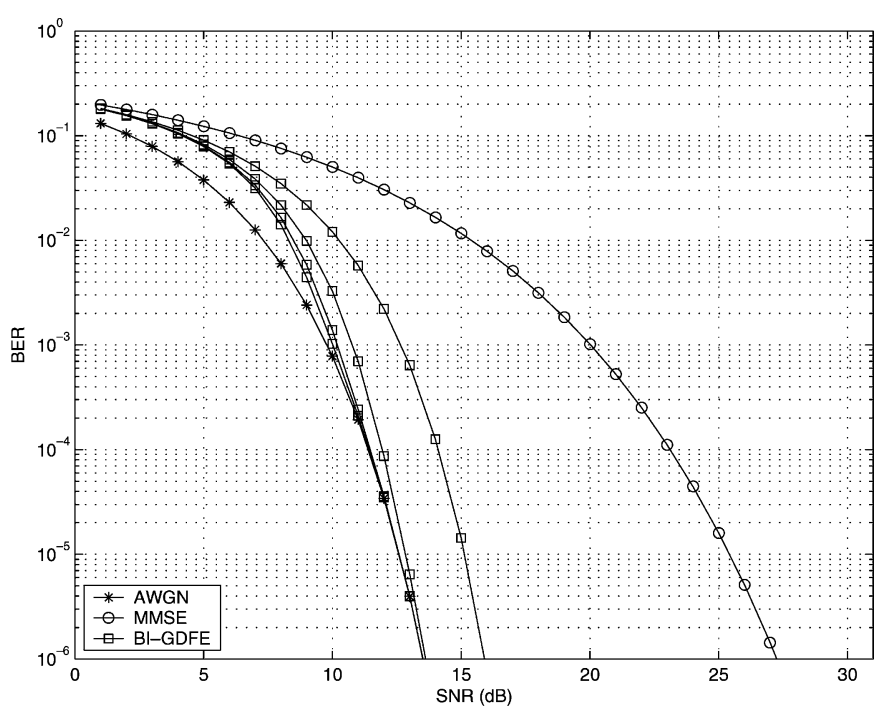

(b)

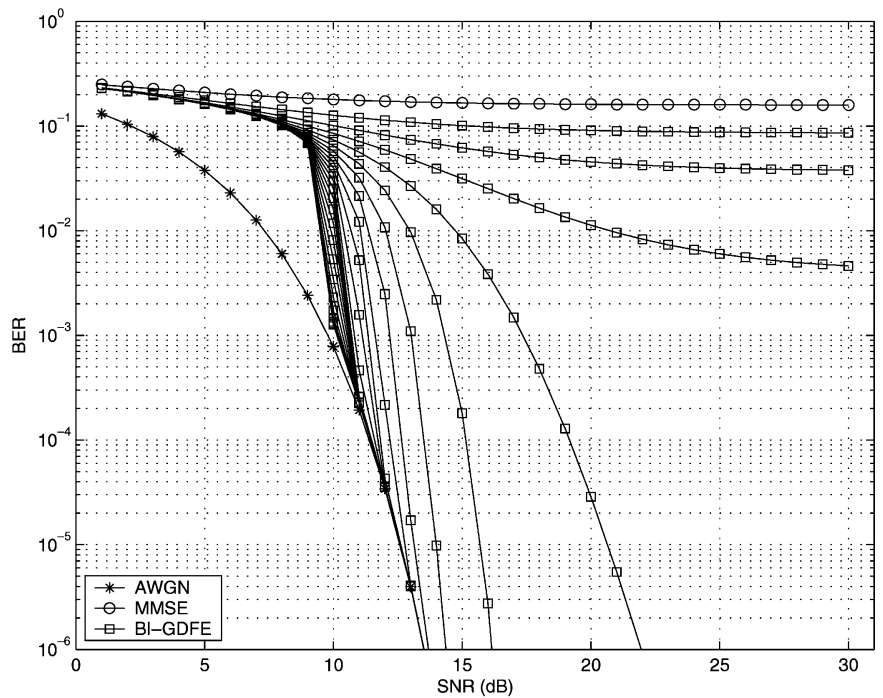

(d)

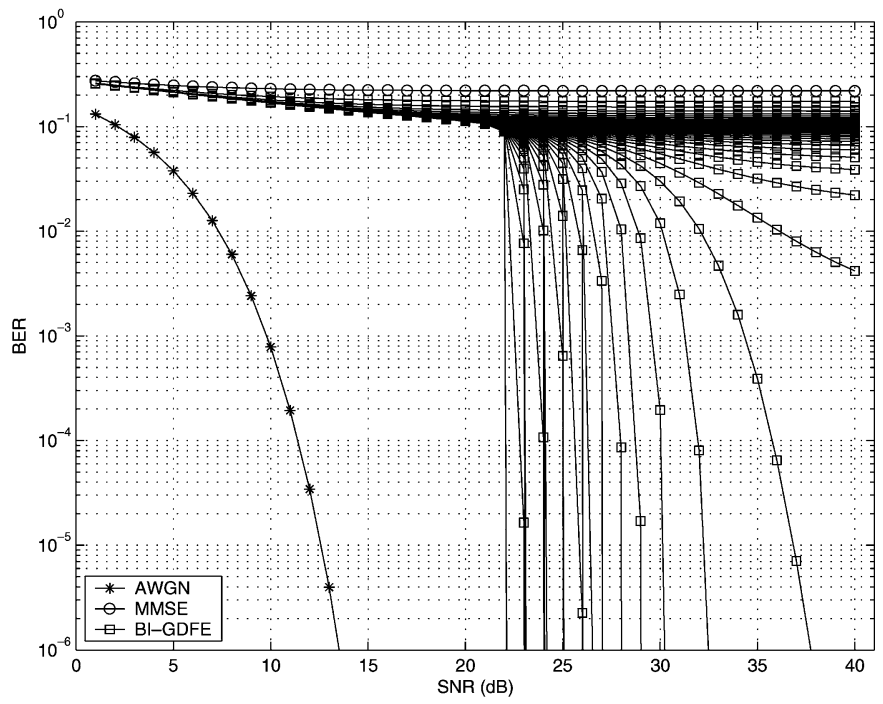

(f)

Fig. 8. Asymptotic performance for BI-GDFE with: (a) $\alpha=0.5,2$ iterations. (b) $\alpha=1$, four iterations. (c) $\alpha=1.5,10$ iterations. (d) $\alpha=2,30$ iterations. (e) $\alpha=2.5,50$ iterations. (f) $\alpha=2.66,80$ iterations. 


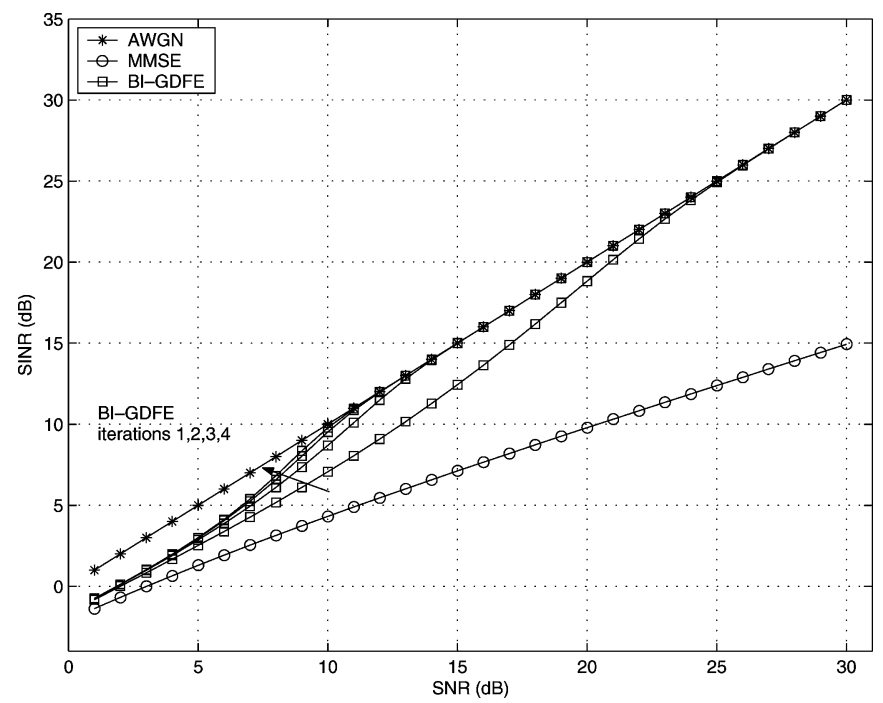

Fig. 9. Dynamics of the output SINRs of the proposed BI-GDFE receiver for $\alpha=1$ and four iterations.

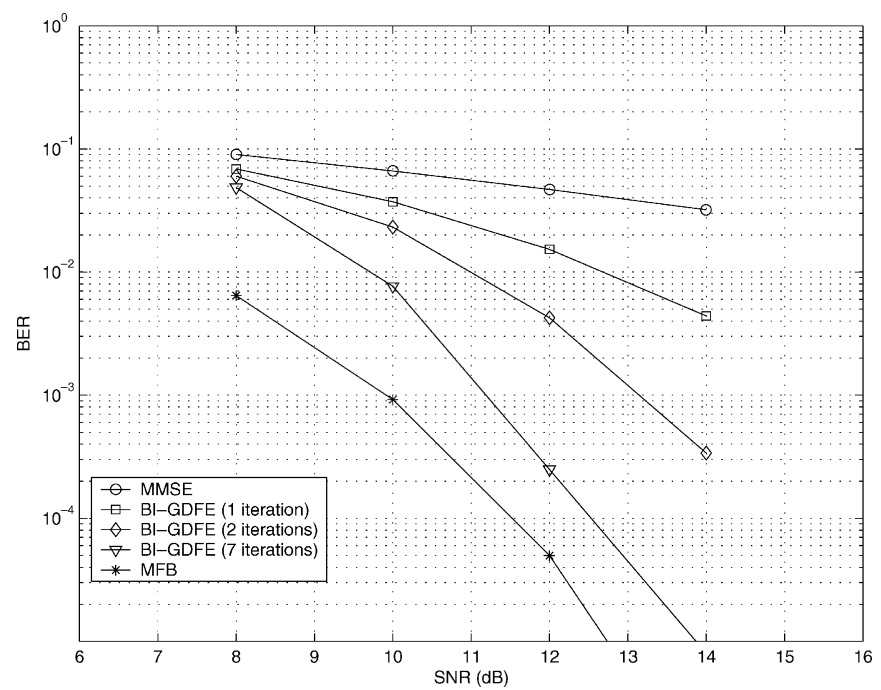

Fig. 10. Performance of MIMO systems using different receiver with $P=$ 100 and $N=90$.

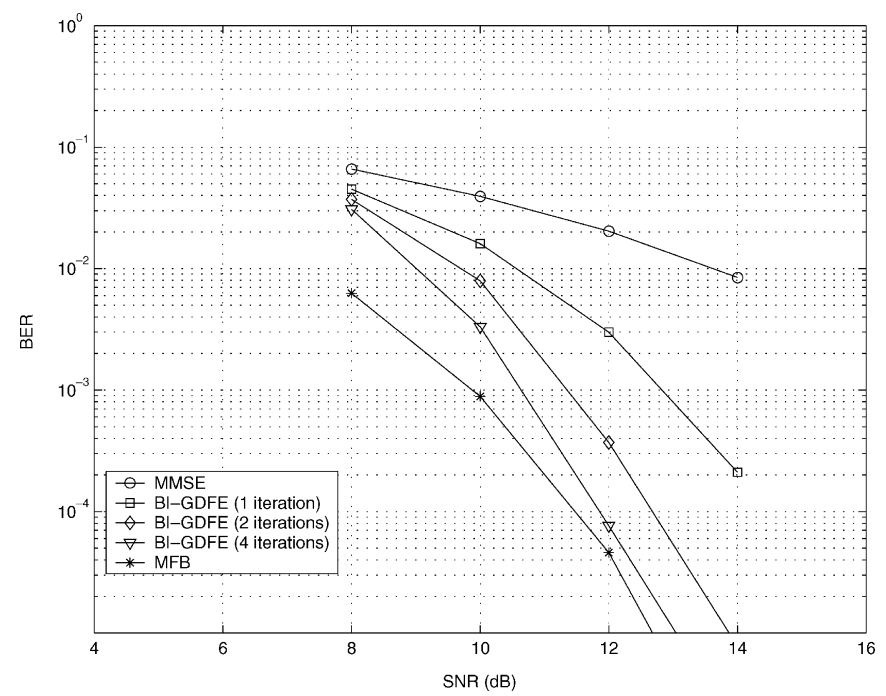

Fig. 11. Performance of MIMO-SCCP systems using different receivers.

\section{APPENDIX A}

We look at the limiting correlation $r_{12}$ when $P \rightarrow \infty, N \rightarrow$ $\infty$ with $P / N \rightarrow$ constant $\alpha$ using random matrix theory. First, use the definition of $\mathbf{H}_{1}$, we have $\mathbf{H} \mathbf{H}^{\mathrm{H}}=\mathbf{H}_{1} \mathbf{H}_{1}^{\mathrm{H}}+\mathbf{h}_{1} \mathbf{h}_{1}^{\mathrm{H}}$. Thus, according to (11),

$$
\begin{aligned}
r_{12}= & \mathbf{h}_{1}^{\mathrm{H}}\left[\mathbf{H}_{1} \mathbf{H}_{1}^{\mathrm{H}}+\left(\frac{1}{\gamma}\right) \mathbf{I}\right]^{-1}\left[\mathbf{H H}^{\mathrm{H}}+\left(\frac{1}{\gamma}\right) \mathbf{I}\right] \\
& \times\left[\mathbf{H}_{2} \mathbf{H}_{2}^{\mathrm{H}}+\left(\frac{1}{\gamma}\right) \mathbf{I}\right]^{-1} \mathbf{h}_{2} \\
= & \mathbf{h}_{1}^{\mathrm{H}}\left[\mathbf{H}_{2} \mathbf{H}_{2}^{\mathrm{H}}+\sigma^{2} \mathbf{I}\right]^{-1} \mathbf{h}_{2} \\
& +\mathbf{h}_{1}^{\mathrm{H}}\left[\mathbf{H}_{1} \mathbf{H}_{1}^{\mathrm{H}}+\sigma^{2} \mathbf{I}\right]^{-1} \mathbf{h}_{1} \mathbf{h}_{1}^{\mathrm{H}}\left[\mathbf{H}_{2} \mathbf{H}_{2}^{\mathrm{H}}+\sigma^{2} \mathbf{I}\right]^{-1} \mathbf{h}_{2} .
\end{aligned}
$$

Using matrix inversion lemma, we notice

$$
\begin{aligned}
\mathbf{h}_{1}^{\mathrm{H}} & {\left[\mathbf{H}_{2} \mathbf{H}_{2}^{\mathrm{H}}+\left(\frac{1}{\gamma}\right) I\right]^{-1} \mathbf{h}_{2} } \\
= & \frac{1}{1+\mathbf{h}_{1}^{\mathrm{H}}\left[\mathbf{H}_{12} \mathbf{H}_{12}^{\mathrm{H}}+\left(\frac{1}{\gamma}\right) I\right]^{-1} \mathbf{h}_{1}} \\
& \cdot \mathbf{h}_{1}^{\mathrm{H}}\left[\mathbf{H}_{12} \mathbf{H}_{12}^{\mathrm{H}}+\left(\frac{1}{\gamma}\right) I\right]^{-1} \mathbf{h}_{2}
\end{aligned}
$$

where $\mathbf{H}_{12}$ denotes the matrix $\mathbf{H}$ with first and second columns being deleted. Thus, we have

$$
\begin{aligned}
& \mathrm{E}\left[\left|\mathbf{h}_{1}^{\mathrm{H}}\left[\mathbf{H}_{2} \mathbf{H}_{2}^{\mathrm{H}}+\left(\frac{1}{\gamma}\right) I\right]^{-1} \mathbf{h}_{2}\right|^{2}\right] \\
\leq & \mathrm{E}\left[\left|\mathbf{h}_{1}^{\mathrm{H}}\left[\mathbf{H}_{12} \mathbf{H}_{12}^{\mathrm{H}}+\left(\frac{1}{\gamma}\right) I\right]^{-1} \mathbf{h}_{2}\right|^{2}\right] \\
= & \mathrm{E}\left[\operatorname{Tr}\left[\mathbf{H}_{12} \mathbf{H}_{12}^{\mathrm{H}}+\left(\frac{1}{\gamma}\right) I\right]^{-1}\right. \\
& \left.\cdot \mathbf{h}_{2} \mathbf{h}_{2}^{\mathrm{H}}\left[\mathbf{H}_{12} \mathbf{H}_{12}^{\mathrm{H}}+\left(\frac{1}{\gamma}\right) I\right]^{-1} \mathbf{h}_{1} \mathbf{h}_{1}^{\mathrm{H}}\right] \\
= & \frac{1}{N^{2}} \mathrm{E}\left[\operatorname{Tr}\left[\mathbf{H}_{12} \mathbf{H}_{12}^{\mathrm{H}}+\left(\frac{1}{\gamma}\right) I\right]^{-2}\right] \leq \frac{1}{N \gamma^{2}}
\end{aligned}
$$

which implies that, when $N \rightarrow \infty$

$$
\mathbf{h}_{1}^{\mathrm{H}}\left[\mathbf{H}_{2} \mathbf{H}_{2}^{\mathrm{H}}+\left(\frac{1}{\gamma}\right) \mathbf{I}\right]^{-1} \mathbf{h}_{2} \rightarrow 0, \quad \text { i.p. }
$$

where i.p. means "convergence in probability." On the other hand, according to [17], [22]

$$
\mathbf{h}_{1}^{\mathrm{H}}\left[\mathbf{H}_{1} \mathbf{H}_{1}^{\mathrm{H}}+\left(\frac{1}{\gamma}\right) I\right]^{-1} \mathbf{h}_{1} \rightarrow \int \frac{1}{x+\left(\frac{1}{\gamma}\right)} d F_{\alpha}(x), \quad \text { i.p. }
$$

where $F_{\alpha}(x)$ is the limiting distribution of the empirical eigenvalue distribution function of random matrix $\mathbf{H H}^{\mathrm{H}}$. It follows that

$$
\begin{aligned}
\mathbf{h}_{1}^{\mathrm{H}}\left[\mathbf{H}_{1} \mathbf{H}_{1}^{\mathrm{H}}+\left(\frac{1}{\gamma}\right) I\right]^{-1} \mathbf{h}_{1} \mathbf{h}_{1}^{\mathrm{H}} & \\
& \times\left[\mathbf{H}_{2} \mathbf{H}_{2}^{\mathrm{H}}+\left(\frac{1}{\gamma}\right) I\right]^{-1} \mathbf{h}_{2} \rightarrow 0 \text {, i.p. }
\end{aligned}
$$

Consequently, we have $r_{12} \rightarrow 0$ in probability. 


\section{APPENDIX B \\ PROOF OF THEOREM 1}

The input signal to the slicer at the $\ell$ th iteration is $\mathbf{z}_{\ell}=$ $\mathbf{K}_{\ell}^{\mathrm{H}} \mathbf{x}+\mathbf{D}_{\ell} \hat{\mathbf{s}}_{\ell-1}$. Letting $\mathbf{A}_{\ell}=\operatorname{diag}\left(\mathbf{K}_{\ell}^{\mathrm{H}} \mathbf{H}\right)$, and noting that $\mathbf{x}=\mathbf{H s}+\mathbf{n}, \mathbf{z}_{\ell}$ can be written as

$$
\mathbf{z}_{\ell}=\mathbf{A}_{\ell} \mathbf{s}+\left(\mathbf{K}_{\ell}^{\mathrm{H}} \mathbf{H}-\mathbf{A}_{\ell}\right) \mathbf{s}+\mathbf{D}_{\ell} \hat{\mathbf{s}}_{\ell-1}+\mathbf{K}_{\ell}^{\mathrm{H}} \mathbf{n} .
$$

Let $\mathbf{C}_{\ell}=\mathbf{K}_{\ell}^{\mathrm{H}} \mathbf{H}-\mathbf{A}_{\ell}$, and $\tilde{\mathbf{n}}_{\ell}=\mathbf{C}_{\ell} \mathbf{s}+\mathbf{D}_{\ell} \hat{\mathbf{s}}_{\ell-1}+\mathbf{K}_{\ell}^{\mathrm{H}} \mathbf{n}$. Then it can be shown that

$$
\begin{aligned}
& \mathbf{R}_{\tilde{n}_{\ell}}=\mathrm{E}\left[\tilde{\mathbf{n}}^{\mathrm{H}}\right]=\sigma_{s}^{2}\left(1-\rho_{\ell-1}^{2}\right) \mathbf{C}_{\ell} \mathbf{C}_{\ell}^{\mathrm{H}} \\
& \quad+\sigma_{s}^{2}\left(\rho_{\ell-1} \mathbf{C}_{\ell}+\mathbf{D}_{\ell}\right)\left(\rho_{\ell-1} \mathbf{C}_{\ell}+\mathbf{D}_{\ell}\right)^{\mathrm{H}}+\sigma_{n}^{2} \mathbf{K}_{\ell}^{\mathrm{H}} \mathbf{K}_{\ell} .
\end{aligned}
$$

In deriving (47), we have used the facts/assumptions that $\mathrm{E}\left[\mathbf{s s}^{\mathrm{H}}\right]=\sigma_{s}^{2} \mathbf{I}, \mathrm{E}\left[\mathbf{s s}_{\ell-1}^{\mathrm{H}}\right]=\rho_{\ell-1} \sigma_{s}^{2} \mathbf{I}$, and $\mathrm{E}\left[\mathbf{s}_{\ell-1} \mathbf{s}_{\ell-1}^{\mathrm{H}}\right]=\sigma_{s}^{2} \mathbf{I}$. The SINR for the $i$ th symbol is then given by

$$
\gamma_{\ell}(i)=\frac{\left|\mathbf{A}_{\ell}(i, i)\right|^{2} \sigma_{s}^{2}}{\mathbf{R}_{\tilde{n}_{\ell}}(i, i)}
$$

Recall that our objective is to preserve the signal component using the FFE, and to cancel out the interference using the FBE as much as possible. In (47), the middle term of the RHS is nonnegative, thus to minimize the effect from both interference and noise, we have to choose

$$
\mathbf{D}_{\ell}=-\rho_{\ell-1} \mathbf{C}_{\ell}=\rho_{\ell-1}\left(\mathbf{A}_{\ell}-\mathbf{K}_{\ell}^{\mathrm{H}} \mathbf{H}\right) .
$$

In this case, the middle term of the RHS of (47) becomes zero matrix, thus SINR for the $i$ th detected symbol is given by

$$
\gamma_{\ell}(i)=\frac{\left|\mathbf{k}_{\ell, i}^{\mathrm{H}} \mathbf{h}_{i}\right|^{2}}{\left(1-\rho_{\ell-1}^{2}\right) \sum_{j \neq i}\left|\mathbf{k}_{\ell, i}^{\mathrm{H}} \mathbf{h}_{j}\right|^{2}+\left(\frac{1}{\gamma}\right)\left\|\mathbf{k}_{\ell, i}\right\|^{2}},
$$

where $\mathbf{k}_{\ell, i}$ is the $i$ th column of $\mathbf{K}_{\ell}$. Intuitively, the FBE cancels out all interferences using the decision-directed symbols, if they are all perfectly detected. The equalized SINR shown in (50) can also be achieved through an equivalent $N$-input $N$ output channel model given by

$$
\mathbf{x}=\mathbf{h}_{i} s(i)+\sqrt{1-\rho_{\ell-1}^{2}} \sum_{j \neq i} \mathbf{h}_{j} s(j)+\mathbf{n} .
$$

The MMSE receiver to maximize $\gamma_{\ell}(i)$ of (50) is given by

$$
\mathbf{k}_{\ell, i}=\left[\mathbf{h}_{i} \mathbf{h}_{i}^{\mathrm{H}}+\left(1-\rho_{\ell-1}^{2}\right) \sum_{j \neq i} \mathbf{h}_{j} \mathbf{h}_{j}^{\mathrm{H}}+\left(\frac{1}{\gamma}\right) \mathbf{I}\right]^{-1} \mathbf{h}_{i} .
$$

The unbiased estimate of $s(i)$ can be derived by multiplying the equalization output with a scaling factor, $\gamma_{\ell}(i) /\left(\gamma_{\ell}(i)-1\right)$ [15]. Applying the equalizer (52) and the scaling, the output can then be utilized for hard decisions for any type of modulations. If the SINR maximization is of interest, we can choose

$$
\mathbf{K}_{\ell}=\left[\left(1-\rho_{\ell-1}^{2}\right) \mathbf{H} \mathbf{H}^{\mathrm{H}}+\left(\frac{1}{\gamma}\right) \mathbf{I}\right]^{-1} \mathbf{H}
$$

This is based on the fact that SINR maximization is independent of the weight norms (as long as they are nonzero). Note that for constant-modulus modulations, hard decisions can be made by equalizing $\mathrm{x}$ using (53) only. Finally, the equalized SINRs for each symbol at the slicer input can be calculated by inserting (49) and (53) into (48). This concludes the proof.

\section{REFERENCES}

[1] E. Agrell, T. Eriksson, A. Vardy, and K. Zeger, "Closest point search in lattices," IEEE Trans. Inf. Theory, vol. 48, no. 8, pp. 2201-2214, Aug. 2002.

[2] M. O. Damen, H. E. Gamal, and G. Caire, "On maximum-likelihood detection and the search for the closest lattice point," IEEE Trans. Inf. Theory, vol. 49, no. 10, p. 2389, 2401, Oct. 2003.

[3] H. Vikalo, B. Hassibi, and U. Mitra, "Sphere-constrained ML detection for frequency selective channels," in Proc. IEEE Int. Conf. Acoustics, Speech, Signal Processing (ICASSP), 2003, pp. IV-1-IV-4.

[4] H. Artes, D. Seetheler, and F. Hlaeatsch, "Efficient detection algorithm for MIMO channels: A geometrical approach to approximate ML detection," IEEE Trans. Signal Process., vol. 51, no. 11, pp. 2808-2820, Nov. 2003.

[5] S. Beheshti, S. H. Isabelle, and G. W. Wornell, "Joint intersymbol and multiple access interference suppression algorithms for CDMA systems," Eur. Trans. Telecomm. Relat. Technol., vol. 9, pp. 403-418, Sep.-Oct. 1998.

[6] A. M. Chan and G. W. Wornell, "A class of block-iterative equalizers for intersymbol interference channels: Fixed channel results," IEEE Trans. Commun., vol. 49, no. 11, pp. 1966-1976, Nov. 2001.

[7] N. Benvenuto and S. Tomasin, "Block iterative DFE for single carrier modulation," Electron. Lett., vol. 38, no. 19, pp. 1144-1145, Sep. 2002.

[8] R. Kalbasi, R. Dinis, D. D. Falconer, and A. H. Banihashemi, "Layered space-time receivers for single carrier transmission with iterative frequency domain equalization," presented at the IEEE Vehicular Technology Conf. (VTC) Spring' 04, Milan, Italy, May 2004.

[9] L. Brunel, "Multiuser detection techniques using maximum likelihood sphere decoding in multicarrier CDMA systems," IEEE Trans. Wireless Commun., vol. 3, no. 3, pp. 949-957, May 2004.

[10] J. G. Proakis, Digital Communications, Third ed. New York: McGrawHill, 1995.

[11] D. N. C. Tse and S. V. Hanly, "Linear multiuser receivers: Effective interference, effective bandwidth and user capacity," IEEE Trans. Inf. Theory, vol. 45, no. 2, pp. 641-657, Mar. 1999

[12] S. Verdú and S. Shamai (Shitz), "Spectral efficiency of CDMA with random spreading," IEEE Trans. Inf. Theory, vol. 45, no. 2, pp. 622-640, Mar. 1999.

[13] G. Ginis and J. M. Cioffi, "On the relation between V-BLAST and the GDFE," IEEE Commun. Lett., vol. 5, pp. 364-366, Sep. 2001.

[14] G. Foschini and M. Gans, "On limits of wireless communications in fading environment when using multiple antennas," Wireless Personal Commun., vol. 8, no. 6, pp. 315-335, 1998.

[15] J. M. Cioffi, Digital Communications, CA: Stanford Univ., 2003, Course Reader.

[16] X. Wang and H. V. Poor, "Blind multiuser detection: A subspace approach," IEEE Trans. Inf. Theory, vol. 44, no. 2, pp. 677-690, Mar. 1998

[17] Z. D. Bai and J. W. Silverstein, "No eigenvalues outside the support of the limiting spectral distribution of large dimensional random matrices," Ann. Probab., vol. 26, pp. 16-345, 1998.

[18] D. Falconer, S. Ariyavisitakul, A. Benyamin-seeyar, and B. Edison, "Frequency domain equalization for single-carrier broadband wireless systems," IEEE Commun. Mag., vol. 4, pp. 58-66, Apr. 2004. 
[19] J. Tubbax, L. Vander Perre, S. Donnay, and M. Engels, "Single-carrier communications using decision-feedback equalization for multiple antennas," presented at the IEEE Int. Conf. Communications (ICC), Anchorage, Ak, May 2003.

[20] R. Kalbasi, R. Dinis, D. Falconer, and A. H. Banihashemi, "Hybrid time-frequency layered space-time receivers for severe time-dispersive channels," presented at the IEEE 5th Workshop Signal Processing Advances in Wireless Communications (SPAWC), Lisbon, Portugal, 2004.

[21] L. Li, A. M. Tulino, and S. Verdu, "Design of reduced-rank MMSE multiuser detectors using random matrix theory," IEEE Trans. Inf. Theory, vol. 50, Jun. 2004.

[22] A. M. Tulino and S. Verdu, "Random matrix theory and wireless communications," in Foundations and Trends in Communications and Information: Now, 2004.

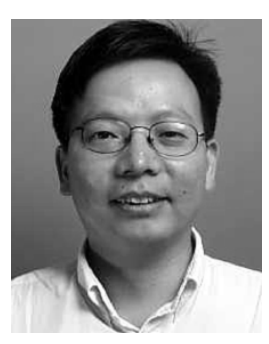

Ying-Chang Liang (SM'00) received the Ph.D. degree in electrical engineering from Jilin University, China, in 1993.

$\mathrm{He}$ is a Lead Scientist with the Institute for Infocomm Research (I2R), Singapore. He also holds Adjunct Associate Professor positions with the Nanyang Technological University and National University of Singapore, Singapore. From December 2002 to December 2003, he was a visiting scholar with the Department of Electrical Engineering, Stanford University, CA. From August 1997 to November 2001, he was a Senior Member of Technical Staff with the Centre for Wireless Communications, Singapore. His research interest includes space-time wireless communications, reconfigurable signal processing and cognitive radio, smart antennas, ultrawideband communications, and capacity-achieving schemes for MIMO fading channels.

Dr. Liang received the Best Paper Award from the 50th IEEE Vehicular Technology Conference. He was also the corecipient of the 1997 National Natural Science Award from China. He has been an Associate Editor for the IEEE TRANSACtions ON Wireless COMmUNicATIONS since 2002. He was Publication Chair for the 2001 IEEE Workshop on Statistical Signal Processing.

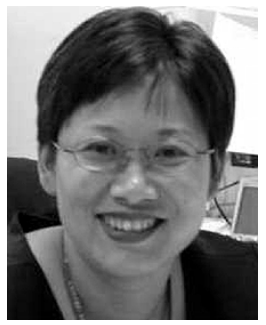

Sumei Sun received the B.Sc. degree from Peking University, China, in 1990, and the M.Eng. degree from Nanyang Technological University, Singapore, in 1996.

She is currently pursuing the Ph.D. degree on a part-time basis at National University of Singapore. She was an Assistant Lecturer with the Electronics Department, Peking University, from July 1990 to June 1993, a Research Assistant with the School of EEE, Nanyang Technological University, from November 1993 to July 1995. Since August 1995, she has been with the Institute for Infocomm Research (previously the Centre for Wireless Communications), and was involved in R\&D projects on CDMA multiuser detection and interference cancellation, 3G W-CDMA downlink terminal algorithms, and broadband wireless access transceivers. Since 2001, she has been leading the MIMO-OFDM effort for high data rate wireless LAN/PAN applications at $\mathrm{I}^{2} \mathrm{R}$. Her research interests include MIMO system architecture, iterative receiver algorithms, and error correction coding.

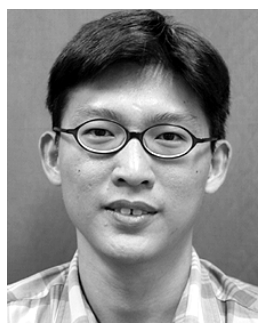

Chin Keong Ho (S'05) received the B.Eng. (firstclass honors) and M. Eng. degrees from the Department of Electrical Engineering, National University of Singapore, in 1999 and 2001, respectively.

$\mathrm{He}$ is currently working toward the Ph.D. degree at Eindhoven University of Technology, Eindhoven, The Netherlands, conducting joint work with Philips Research. He was with the Institute for Infocomm Research, Singapore, from August 2000 to October 2004. His research interest lies in signal processing and wireless communications for multicarrier and 